NISTIR 7961

\title{
Processes Analytics Formalism for Decision Guidance in Sustainable Manufacturing
}

Alexander Brodsky

Guodong Shao

Frank Riddick

http://dx.doi.org/10.6028/NIST.IR.7961

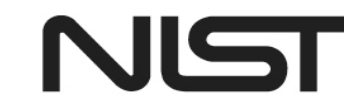

National Institute of Standards and Technology U.S. Department of Commerce 
NISTIR 7961

\title{
Processes Analytics Formalism for Decision Guidance in Sustainable Manufacturing
}

\author{
Alexander Brodsky \\ George Mason University \\ Guodong Shao \\ Frank Riddick \\ System Integration Division \\ Engineering Laboratory
}

http://dx.doi.org/10.6028/NIST.IR.7961

November 2013

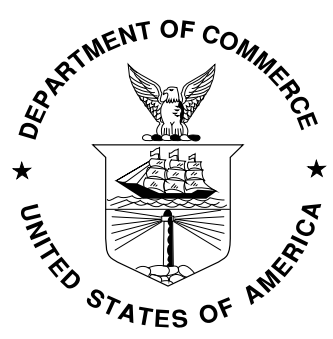

U.S. Department of Commerce

Penny Pritzker, Secretary

National Institute of Standards and Technology Patrick D. Gallagher, Under Secretary of Commerce for Standards and Technology and Director 


\section{TABLE OF CONTENTS}

1. INTRODUCTION

2. The needs for SUSTAINABLE process analytics formalism

3. The concept of sustainable process analytics formalism

3.2 SPAF concept through a manufacturing example

3.3 Structures of SPAF

3.4 Potential users of the SPAF

4. Illustrative example using SPAF

A. SPAF model syntax

B. SPAF formal semantics

C. SPAF Query Computation 


\begin{abstract}
This paper introduces NIST's Sustainable Process Analytics Formalism (SPAF) to facilitate the use of simulation and optimization technologies for decision support in sustainable manufacturing. SPAF allows formal modeling of modular, extensible, and reusable process components and enables sustainability performance prediction, what-if analysis, and decision optimization based on mathematical programming. SPAF models describe (1) process structure and resource flow, (2) process data, (3) control variables, and (4) computation of sustainability metrics, constraints, and objectives. This paper presents the SPAF syntax and formal semantics, provides a sound and complete algorithm to translate SPAF models into formal mathematical programming models, and illustrates the use of SPAF through a manufacturing process example.
\end{abstract}




\section{INTRODUCTION}

To be successful in today's complex, rapidly changing, and highly competitive world, manufacturers must begin using sustainable practices throughout their manufacturing operations. The United States Department of Commerce (DOC) identifies Sustainable Manufacturing (SM) as one of its high-priority performance goals, defining SM as the "creation of manufactured products that use processes that minimize negative environmental impacts, conserve energy and natural resources, are safe for employees, communities, and consumers, and are economically sound” (DOC 2010).

Increasingly, some large companies are making efforts to make their operations and manufacturing processes more sustainable (Fujitsu 2011, GM 2010, Rockwell Automation 2010). However, most of these projects are customized and conducted on a piecemeal basis. The solutions are normally not easily reusable and not easily extensible. The effect of many complex interactions is often overlooked. Furthermore, most of the small and medium-sized enterprises (SMEs) lack the capability to manage energy and material efficiency in a systematic, quantitative, and optimal manner required to meet their sustainability goals. To address these challenges, a standard formal methodology is needed to model, exchange, and reuse manufacturing process knowledge for effective sustainability performance analysis.

This is the focus of this paper. A Sustainable Process Analytics Formalism (SPAF) has been developed at the National Institute of Standards and Technology (NIST) to facilitate the use of simulation and optimization technologies for decision support in sustainable manufacturing. SPAF allows formal modeling of modular, extensible and reusable process components and enables sustainability performance prediction, what-if analysis and decision optimization based on mathematical programming. More specifically, the contributions of this paper include (1) the concept of SPAF that enables formal representation of sustainable process structure and resource flow, data, control parameters, metrics, and constraints; (2) the syntax and formal semantics of SPAF; (3) a sound and complete algorithm to translate SPAF models into formal mathematical programming models; and (4) a sustainable manufacturing example that illustrates SPAF.

The rest of this paper is organized as follows. Section 2 briefly discusses the needs for the SPAF; section 3 introduces the context and concept of SPAF and potential users; section 4 explains the SPAF using an example; in section 5, a summary is provided and future work are discussed. Finally, the appendix presents the detail SPAF syntax and semantics.

\section{THE NEEDS FOR SUSTAINABLE PROCESS ANALYTICS FORMALISM}

Formal description and representation of sustainable processes also provides a basis for standardization. Such standardization in turn is the foundation for system integration, process analysis, and decision optimization, all are essential to the improvement of decision-making on factory floors (NIST 2010, Tanzil and Beloff 2006, NRC 1999). Complex sustainability analysis requires formal simulation (e.g., Delmia Quest) or optimization models (e.g., A Modeling Language for Mathematical Programming (AMPL)) (Berglund et al. 2011, AMPL 2011). Modeling and optimization have been identified as a key enabler for improving SM in the future (SMLC 2011), but require significant modeling expertise and a substantial development effort. SPAF eases the modeling process by providing standard description and tools associated with it, so that the availability, use, and effectiveness of modeling and optimization technologies can be increased. Currently, different analysis tools such as simulation, optimization, and database query languages require different data representation and mathematical abstractions for modeling. Thus, even for the same manufacturing process, the knowledge needs to be represented differently multiple times, rather than just once. This makes model development, modification, and extension very difficult. SPAF facilitates the increase of model reuse. In summary, SPAF is designed to help companies, especially for SMEs, overcome the following major challenges: (1) lack of modeling and 
operation research expertise and (2) duplication of modeling efforts. To satisfy manufacturers' needs, we have decided to include the following desirable features for SPAF:

Data manipulation and querying: SPAF supports data storage, manipulation, and querying. For example, given a model of a specific milling machine, users may want to query the machine specification data provided by the vendor.

What-if analysis: SPAF supports what-if analysis by computing a range of sustainability metrics as a function of non-controllable parameters and control variables in manufacturing processes, based on the formal representation of manufacturing processes and sustainability metrics. For example, given a particular setup of a milling machine, users may want to compute the energy consumption for that setup.

Decision optimization: SPAF enables the formulation of optimization problems for deriving the best option among all alternatives of the operational setting of machines, production plan, and investment options. For example, given a model of a specific milling machine, users may want to find out a setting of the machine that produces the required part while minimizing the energy consumption.

Unified modeling for different tasks: SPAF allows the sustainable process knowledge being represented once, used many times, for different analyses such as data query, what-if analysis, and decision optimization. Figure 1 shows the comparison between the current modeling approach (left hand side of the Figure) and the unified SPAF modeling approach (right hand side of Figure 1). In current modeling approaches, duplicated modeling efforts are needed even for the same manufacturing problem for different kinds of analysis tasks. For example, simulation, optimization, and Life Cycle Assessment (LCA) of a machining process have totally different abstractions and modeling methods. They are independent of each other. However, the unified SPAF modeling approach enables modeling the machining process using SPAF once, and the same model then can be used for data query, what-if analysis, and decision optimization.

Built-in support for process modeling and sustainability metrics: SPAF provides modeling capability for hierarchical composition of processes and resource flows. Representation of sustainable metrics such as $\mathrm{CO}_{2}$ emissions, energy and material consumption, and cost are stored in a model library for reuse.

Modular, extensible, and reusable models: SPAF enables modular model design and creation of model libraries. Modular model design provides definitions for structuring process knowledge into discrete, scalable, and reusable modules consisting of isolated, self-contained functional components and linking these components through well-defined interfaces. A model library stores these model components. Model components in a library can be used as building blocks to formulate new SPAF models for different problems. For example, the users should be able to compose a machine shop model from a number of machine and assembly model components in the library.

Ease of use: SPAF makes process analysis modeling more intuitive and straightforward for domain users such as manufacturing engineers or decision makers. Composing a bigger model using existing model components in a library should be an easy process. No extensive programming, mathematics, operation research, and optimization knowledge is required. The task could be simply dragand-drops if a graphical user interface is developed. 


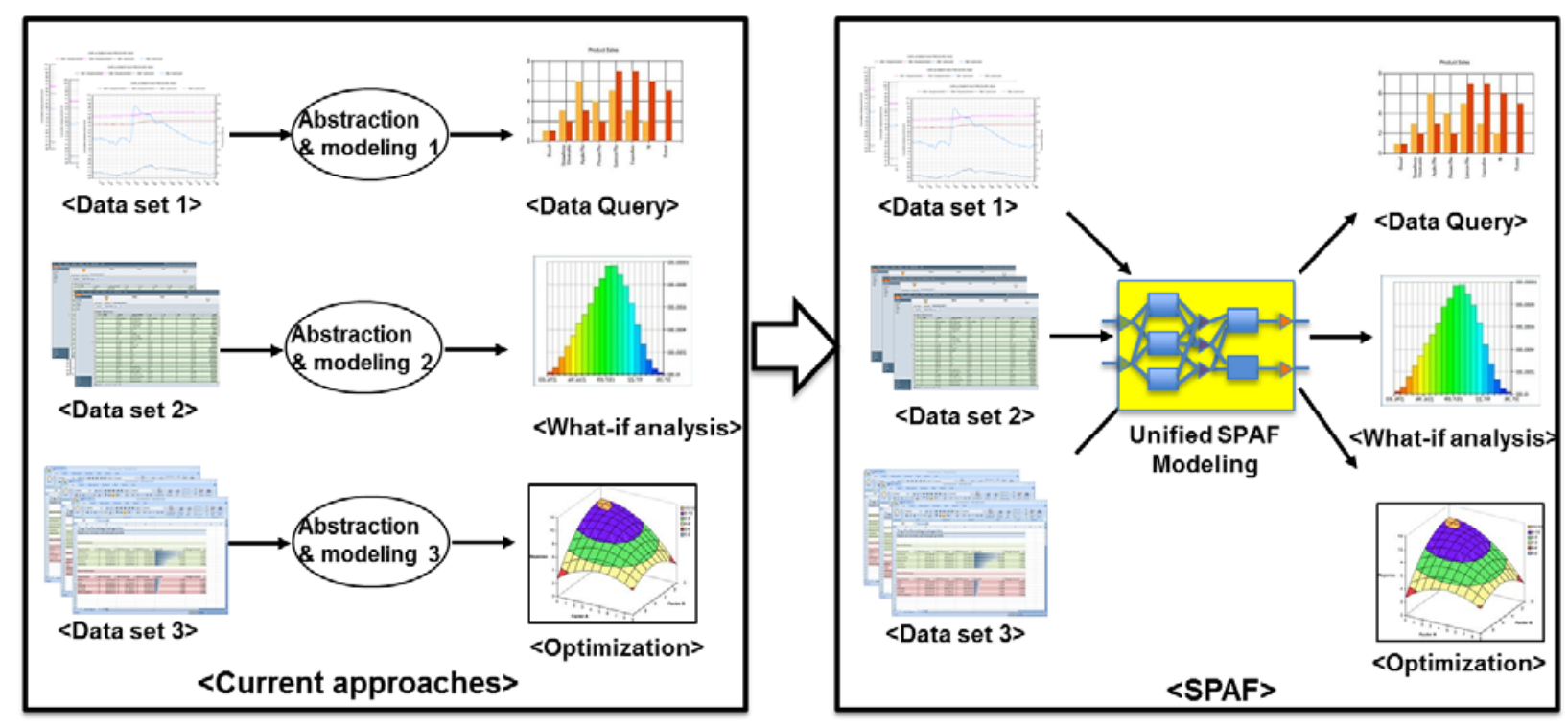

Figure 1 Current modeling approach and the unified SPAF modeling approach

To better understand the SPAF requirements, a variety of modeling languages and formalisms, listed in Table 1, have been analyzed from the perspective of the desirable features and functionalities discussed above. These languages and formalism are selected because of their suitability for at least one of the features discussed above. They include:

- Process description languages such as Process Specification Language (PSL) (ISO 2004), Business Process Model and Notation (BPMN) (OMG, 2010), and Systems Modeling Language (SysML) (OMG, 2012).

- Database query languages such as Structured Query Language (SQL) (ISO 2011) and Extensible Markup Language (XML) Query (XQuery).

- Simulation languages such as SIMAN - a general-purpose SIMulation ANalysis program for modeling combined discrete-continuous systems (Pegden et al. 1995) and Object-Oriented (OO) languages.

- Optimization languages such as AMPL (AMPL, 2011), The General Algebraic Modeling System (GAMS) (GAMS, 2010), and Optimization Programming Language (OPL) (IBM, 2012).

- Non-deterministic optimization semantics for corresponding formalism, which is used for CoJava (Brodsky \& Nash, 2005) and Decision Guidance Query Language (DGQL) (Brodsky \& Wang, 2008).

Process description languages are designed for process description and modeling with a modular, extensible, and reusable approach and can be easy to use via a graphical user interface. The SysML parametric models support mathematical expression for (e.g., performance constraints) the system being designed and provide a foundation for what-if analysis. However, they do not support direct data manipulation and querying, optimization, and unified modeling of different tasks.

Database query languages are specifically designed for data manipulation and querying. They are relatively easy to use, SQL-like skills are sufficient for problem modeling. However, they only allow some limited what-if analysis and optimization for what can be expressed. There is no unified modeling of different tasks. These languages do not have built-in process and sustainability metrics modeling, they are not easily reusable. 
Simulation languages are excellent for what-if analysis. Some simulation tools support process modeling and have user-friendly graphical user interfaces. A few of them even started to support sustainability modeling, e.g., Witness (Waller, 2012). In most cases, simulation languages support modular, extensible, and reusable modeling. However, they are not the appropriate tools for data querying and optimization. Optimization by simulation approach is time-consuming and the results may not be as accurate as those derived by using optimization tools. There is no unified modeling capability for different tasks discussed above. Basic simulation modeling of processes requires object-oriented programming skills that most manufacturing or process engineers do not have.

Optimization languages are designed for optimization modeling. Some optimization languages such as OPL provide basic support for data manipulation and querying. However, they are not designed for whatif analysis and do not provide unified modeling capability. There is no built-in support for process and sustainability modeling. Current optimization modeling languages are not developed for reuse and modular model construction. Mathematical and optimization modeling skills are required to use them.

Optimization semantics for $\mathrm{OO}$ programming and database query languages are developed to provide features such as data manipulation and querying, what-if analysis, optimization, and unified modeling of these different tasks. However, there is no built-in support for process and sustainability metrics modeling, even though it potentially can be built on top of CoJava (Brodsky and Nash, 2005), which requires Java programming skills. On the other hand, DGQL (Brodsky and Wang, 2008) is relatively easy to use, just like SQL.

SPAF is designed to allow data querying, what-if analysis, optimization, and unified modeling of these different tasks. SPAF provides built-in support for process and sustainability metrics modeling with a components' library. SPAF also supports modularity, extensibility, reusability, and ease of use especially, with a graphical interface. However, the modeling effort will be similar to OPL for new process model components if there is no model library.

Table 1 A comparison table of SPAF and other languages

\begin{tabular}{|c|c|c|c|c|c|c|}
\hline $\begin{array}{c}\text { Model } \\
\text { Languages }\end{array}$ & $\begin{array}{l}\text { Process } \\
\text { Description } \\
\text { Languages } \\
\text { (PSL, } \\
\text { BPMN, } \\
\text { SysML) }\end{array}$ & $\begin{array}{l}\text { Database } \\
\text { Query } \\
\text { Languages } \\
\text { (SQL, } \\
\text { XQuery) }\end{array}$ & $\begin{array}{l}\text { Simulation } \\
\text { Languages } \\
\text { (SIMAN, OO } \\
\text { languages) }\end{array}$ & $\begin{array}{l}\text { Optimization } \\
\text { Modeling Languages } \\
\text { (e.g., AMPL, GAMS, } \\
\text { OPL) }\end{array}$ & $\begin{array}{l}\text { Optimization } \\
\text { Semantics for } \\
\text { OO and Query } \\
\text { Lang's (CoJava, } \\
\text { DGQL) }\end{array}$ & $\begin{array}{l}\text { Design goal for } \\
\text { SPAF }\end{array}$ \\
\hline $\begin{array}{l}\text { Data } \\
\text { manipulation } \\
\text { and querying }\end{array}$ & $\begin{array}{c}\text { Not } \\
\text { directly }\end{array}$ & Yes & $\begin{array}{c}\text { Require } \\
\text { modeling and } \\
\text { programming }\end{array}$ & $\begin{array}{l}\text { AMPL and GAMS are } \\
\text { not designed for } \\
\text { query processing; } \\
\text { OPL has some built- } \\
\text { in support }\end{array}$ & Yes & Yes \\
\hline $\begin{array}{l}\text { What-if } \\
\text { analysis }\end{array}$ & $\begin{array}{l}\text { Process } \\
\text { structure } \\
\text { and flow } \\
\text { etc, not } \\
\text { analytics }\end{array}$ & $\begin{array}{l}\text { Limited (only } \\
\text { what can be } \\
\text { expressed as } \\
\text { DB queries) }\end{array}$ & Yes & No & Yes & Yes \\
\hline Optimization & No & $\begin{array}{l}\text { Limited and } \\
\text { not efficient }\end{array}$ & $\begin{array}{l}\text { Limited and } \\
\text { not efficient }\end{array}$ & Yes & Yes & Yes \\
\hline $\begin{array}{l}\text { Unified } \\
\text { modeling for } \\
\text { different tasks }\end{array}$ & No & No & No & No & Yes & Yes \\
\hline Built-in support & Can be & Can be & Can be built on & No & Can be & Yes with a \\
\hline
\end{tabular}




\begin{tabular}{|l|c|c|c|c|c|c|}
\hline $\begin{array}{l}\text { for process } \\
\text { modeling and } \\
\text { sustainability } \\
\text { metrics }\end{array}$ & extended & extended & top & extended & $\begin{array}{c}\text { components } \\
\text { library }\end{array}$ \\
\hline $\begin{array}{l}\text { Modular, } \\
\text { extensible, and } \\
\text { reusable }\end{array}$ & Yes & $\begin{array}{c}\text { Does not } \\
\text { support OO } \\
\text { extensibility }\end{array}$ & Yes & $\begin{array}{c}\text { Difficult to reuse } \\
\text { models }\end{array}$ & $\begin{array}{c}\text { CoJava - Yes; } \\
\text { DGQL - just like } \\
\text { SQL }\end{array}$ & $\begin{array}{c}\text { Yes with a } \\
\text { components } \\
\text { library }\end{array}$ \\
\hline $\begin{array}{l}\text { Ease of use (by } \\
\text { manufacturing } \\
\text { and business } \\
\text { users) }\end{array}$ & $\begin{array}{c}\text { Can be } \\
\text { easy via } \\
\text { graphical } \\
\text { interface }\end{array}$ & $\begin{array}{c}\text { Relatively } \\
\text { easy (SQL } \\
\text { skills) }\end{array}$ & $\begin{array}{c}\text { Programming } \\
\text { skills to model } \\
\text { analytics; } \\
\text { Many allow } \\
\text { high-level } \\
\text { composition } \\
\text { functionality }\end{array}$ & $\begin{array}{c}\text { Math/optimization } \\
\text { modeling skills }\end{array}$ & $\begin{array}{c}\text { CoJava } \\
\text { (programming } \\
\text { skills); } \\
\text { DGQL (SQL } \\
\text { skills) }\end{array}$ & $\begin{array}{c}\text { Easy for } \\
\text { composite } \\
\text { process, esp. if a } \\
\text { graphical } \\
\text { interface is } \\
\text { added; similar } \\
\text { to OPL for } \\
\text { atomic process } \\
\text { models }\end{array}$ \\
\end{tabular}

\section{THE CONCEPT OF SUSTAINABLE PROCESS ANALYTICS FORMALISM}

\subsection{Context of SPAF}

To explain the context of SPAF, a five-stage SM improvement methodology is depicted in Figure 2. This methodology is based on the ideas of the Six Sigma DMAIC (Define, Measure, Analyze, Improve, and Control) methodology (Chieh, 2010). The methodology proceeds through the following stages:

- Stage 1 - High-Level Assessment: Each factory assesses its sustainability level and status, defines high-level sustainability goals, and identifies areas for improvement regarding its organizational sustainability performance (for both its processes and facilities).

- Stage 2 - Problem Identification and Data Collection: To address areas of improvement identified in Stage 1, more specific case scenarios need to be defined. Modeling objectives, constraints, metrics, and control variables related to each case scenario need to be identified. Relevant data, both manufacturing process- and sustainability- related information, need to be measured, collected, and/or estimated. In reality, process- and sustainability- related data are not always available and when they are, they may exist in various forms, and would typically not yet be formalized.

- Stage 3 - Formal Process Modeling and Data Representation: To prepare for formal analysis and optimization modeling, case scenarios defined in Stage 2 need to be formally described, data collected need to be formally represented, and inputs and controls need to be modeled in a way so that the values of decision variables could be instantiated.

- Stage 4 - Decision Guidance through What-if Analysis and Decision Optimization: The formal process modeling and data representation completed in Stage 3 need to be translated into models that can be solved by commercial off-the-shelf (COTS) tools. Different tasks such as data querying, what-if analysis, and decision optimization will be performed for evaluation and analysis purposes. The analyses provide actionable recommendations to decision makers for improvement implementation.

- Stage 5 - Implementation/Execution: Decision makers can implement and execute the actionable recommendations derived from Stage 4 for sustainability improvement. Occasionally, the evaluation in the previous stage may determine that the goals cannot be achieved using the identified alternative and hence this implementation stage may involve abandoning the now determined-to-be flawed improvement plan. In either case, upon completion of Stage 5, users can continue to the next iteration of the continuous improvement cycle. 
The SPAF supports this methodology at stages 3 and 4 in Figure 2. Note that the formal process modeling and data representation is done uniformly and only once for both what-if analysis and decision optimization as shown in the right hand side of Figure 1. The SPAF models enable decision makers to ask questions in the form of queries that provide computation and optimization solutions as actionable recommendations. SPAF queries include:

1. Process data queries that resemble typical database queries and can be asked directly against the explicit data.

2. What-if analysis queries to compute certain metrics for different scenarios based on available input information.

3. Decision optimization queries to find the best one (minimum or maximum as required) out of all alternatives that satisfy the constraints by using decision variables.

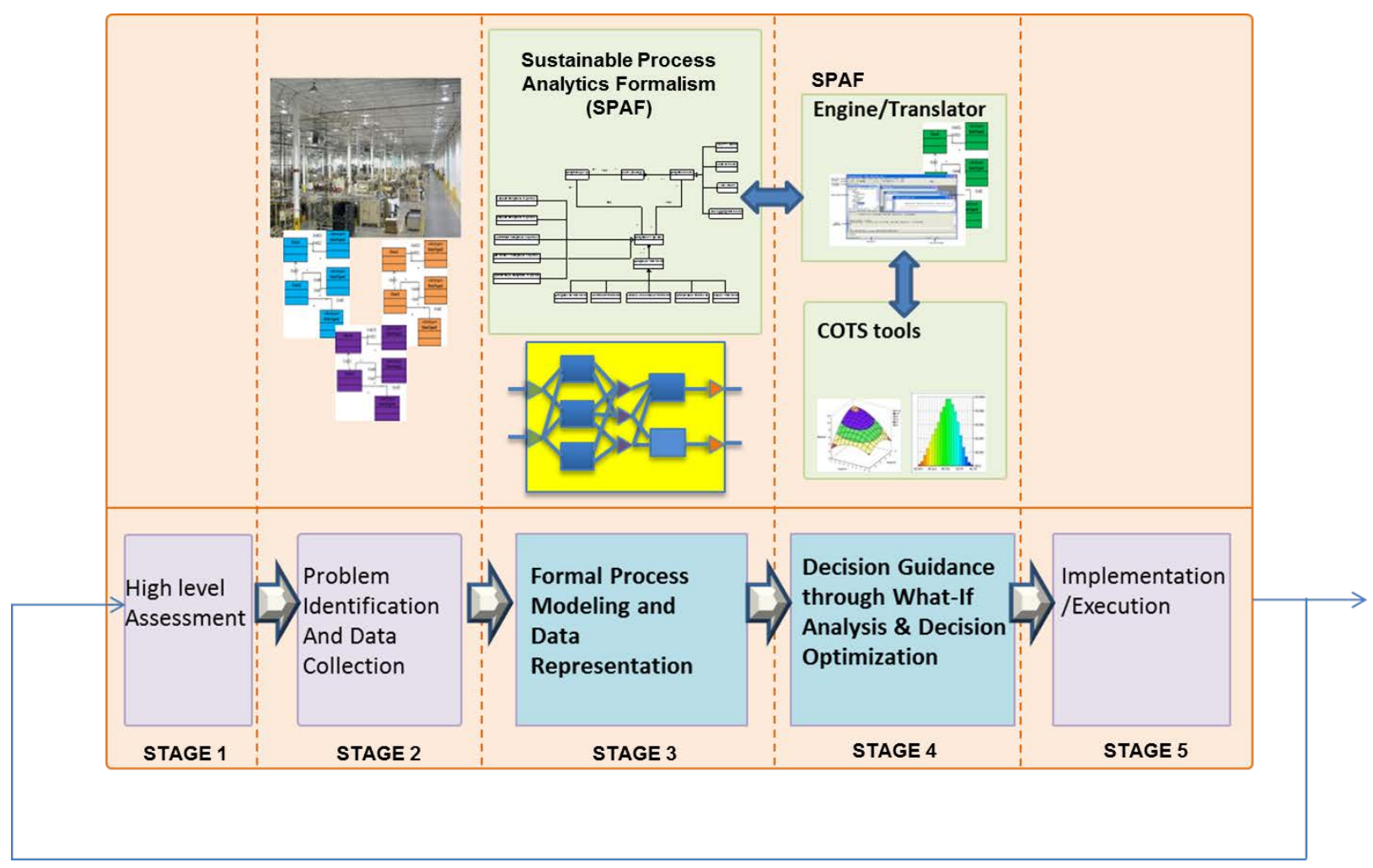

Figure 2 A model-based SM improvement methodology with SPAF

\subsection{SPAF concept through a manufacturing example}

The detailed SPAF syntax and formal semantics will be presented as an appendix. In this subsection, the concept of SPAF modeling is illustrated using an example of a manufacturing process. Assuming we have decided to analyze and optimize sustainability performance for a manufacturing process and collected data for the study. We need to first describe formally the process using SPAF and then solve the problem. The manufacturing process, depicted in Figure 3, has five sub-processes, three machining processes and two assembly processes. The composite process (large rectangle), the sub-processes (small rectangles), flows (lines), and flow aggregators (triangles) are depicted. Two parts, Part 1 and Part 2, provide input for 
the three machining processes. The machining processes produce three intermediate components, Comp 1, Comp 2, and Comp 3. The components produced by the machines, A to C, flow to the assembly processes to be assembled into final products, Product 1 and Product 2. In this example, metrics that can be used to describe the composite process are cost and $\mathrm{CO}_{2}$ emissions. In this example, three specific kinds of questions decision makers pose may include:

1. Process data questions, e.g., what is the maximum capacity of Machine A? How many of Product 2 needs to be produced over a scheduled week?

2. What-if analysis questions, e.g., what are the total cost, energy consumption, and $\mathrm{CO}_{2}$ emissions for a scheduled weekly production under a particular production plan?

3. Decision optimization questions, e.g., how should production plans be set for the machines, the assembly stations, and the flow distributions among them so that the scheduled weekly production can be met within the weekly $\mathrm{CO}_{2}$ cap and at a minimal cost?

To answer these questions, the process structure, flow, sub-process relationships, and associated data need to be clearly understood; and the objective, metrics, constraints, and control variables need to be identified. The models can be expressed with identified data and variables and metrics computation expressions. Optimization models can be formulated with constraints and objectives. The detail SPAF modeling will be discussed in Section 4.

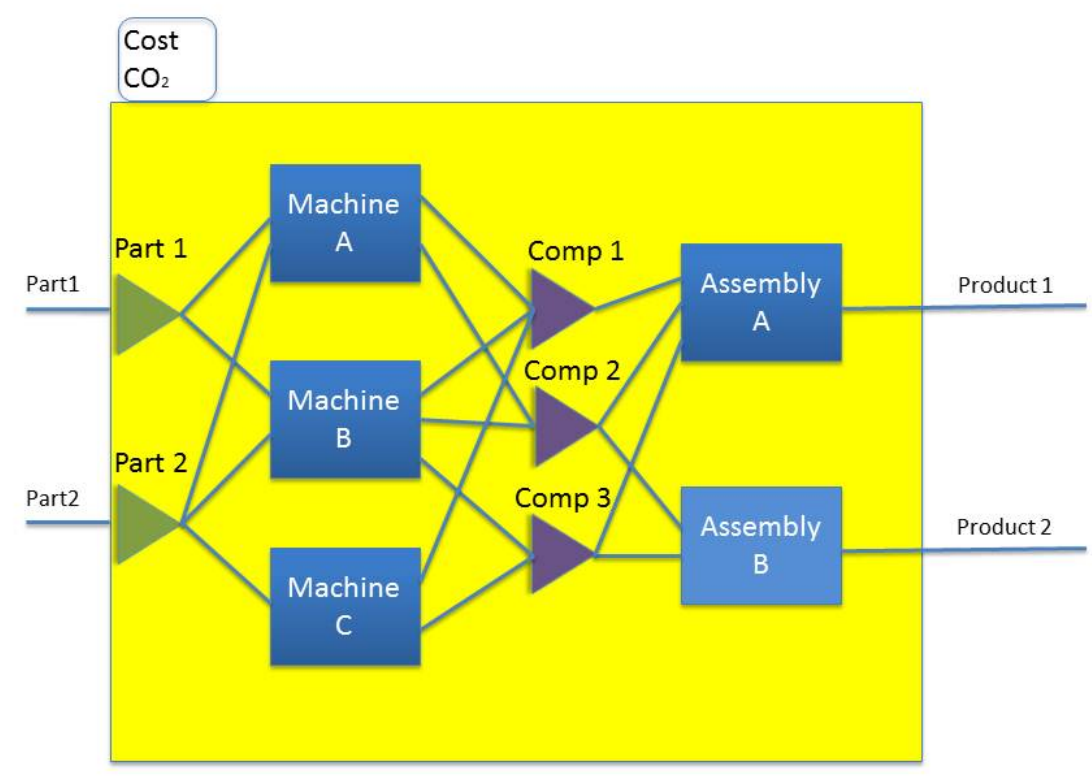

Figure 3 An example: a two-product-manufacturing process for SPAF modeling

\subsection{Structures of SPAF}

The goals of SPAF development are as follows. On the one hand, SPAF needs to be sufficiently expressive for the SM key performance indicators such as energy and material consumption, emission, and cost in industrial scenarios. On the other hand, the formalism needs to be simple for ease of use, which means that high-level abstraction needs to be used. Relevant industry-accepted languages, standards, and tools should be used. The SPAF models are human and machine readable, ready for database storage, translator development, and exchange among modelers/users/systems. 
The SPAF includes two major parts: (1) Generic analytics language - represents generic analytics knowledge and (2) Process description and sustainability metrics model templates - supports sustainable process modeling and sustainability metrics computation.

Figure 4 presents a class diagram for the generic part of the SPAF to describe the structure of the components and their relationships. The components are explained as follows:

- An analytical statement may be assignments, constraints, or decision variable declarations.

- An analytical sequence is a sequence of analytical statements. SPAF analytical sequences can be an explicit, implicit, constraints, alternatives, or optimization analytical sequence to satisfy the different modeling needs.

- Explicit analytical sequence provides all the required process structure and data in the form of assignment statements that assign a data value to each variable in the sequence.

- Implicit analytical sequence extends an explicit analytical sequence with assignment statements that assign an expression (which computes a value from previously defined variables) to a new variable.

- Constraint analytical sequence extends implicit analytical sequence with constraint expressions in terms of previously defined variables.

- Alternative analytical sequence extends a constraint analytical sequence with declaration of variables that do not have an assigned value. Intuitively, an alternative sequence defines a set of feasible computations, corresponding to all possible assignment of values into the declared variables that satisfy the constraints.

- Optimization analytical sequence is an alternatives analytical sequence with the added optimization directive "minimize” or "maximize.” Intuitively, it states the optimization problem of finding an instantiation of values into declared variables that satisfy all the constraint statements, and minimizes/maximizes the indicated objective.

- An analytical model is an (non-optimization) analytical sequence with the added model name and a possible parameter Id.

- A model package is a set of analytical models. Intuitively, it serves as a reusable repository of analytical knowledge, which could be used for different applications.

- An analytical query is a pair of an analytical sequence and a model package that includes all models that are referred to, directly or indirectly, from the process model. 


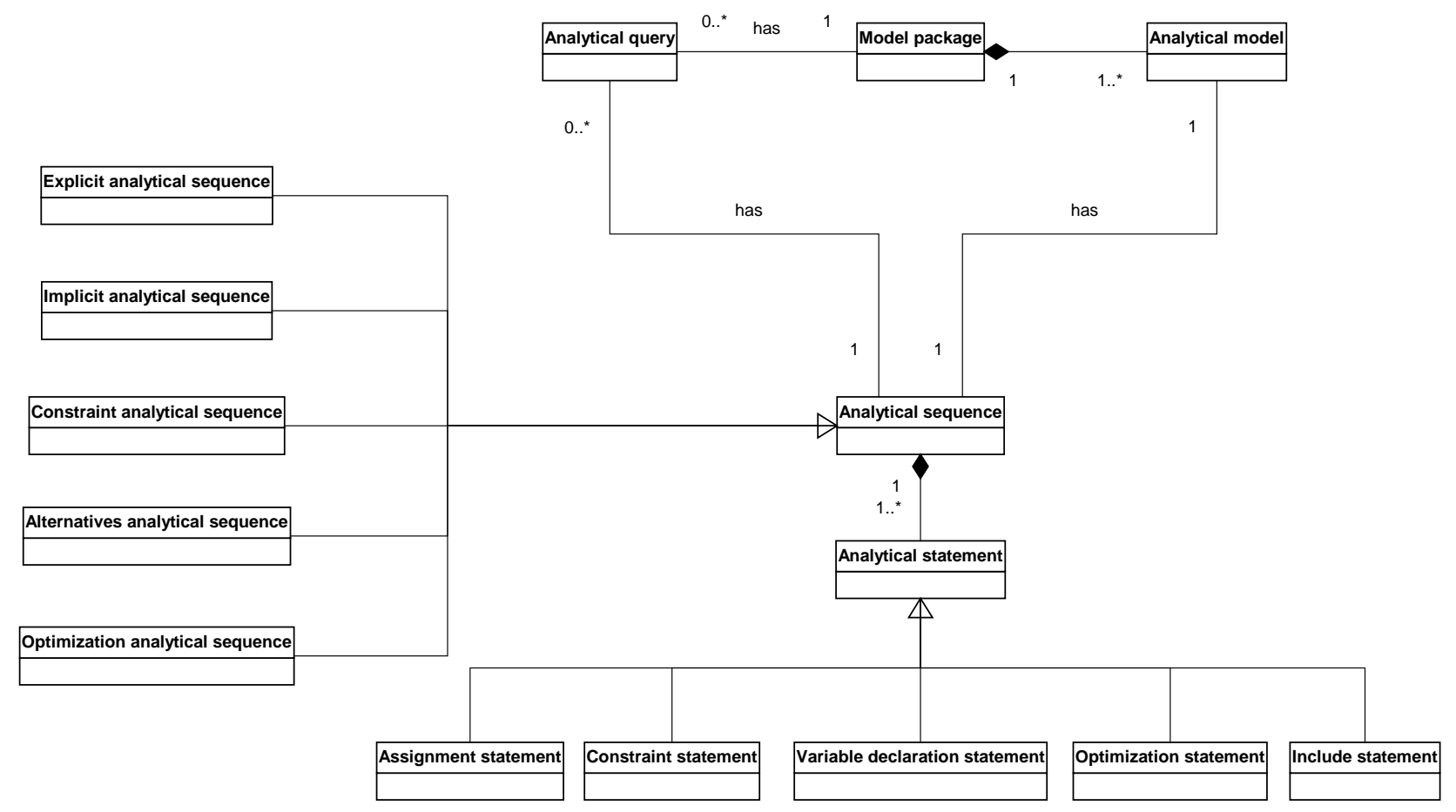

Figure 4 SPAF class diagram - generic analytics language

Figure 5 shows a hierarchical diagram for the process description and sustainability metrics model templates part of the SPAF. For the purpose of formal process representation, four different SPAF model components have been defined. These model components include context, flow, flow aggregator, and process. Context describes data that are globally accessible by all model components; context model includes context ID and associated data attributes. Flow describes entities that physically flow into and out of a process. Flow aggregator aggregates multiple sources of the same type of flows and distributes the outputs as inputs to the other processes. The sum of all inputs of an aggregator must equal the sum of its outputs. A process can be a composite process or a sub-process. The attributes of each component are shown in Figure 6. The process description and sustainability metrics model templates part includes syntax of the SPAF analytical models such as process model, flow model, flow aggregator model, and context model. These analytical models must adhere to a more specialized structure. Figure 5 and Figure 6 are connected through the analytical model.

A process model may be a generic process model or a specific process model. A generic process model can be stored in a model library and reused for developing specific process models. Flow and flow aggregator models may be those for discrete flows, continuous flows, or batch flows. Sustainability metric aggregator models are specifically designed for sustainability metric aggregation for environmental indicators such as energy, emission, material, and waste and economic indicators such as investment, revenue, cost, and return on investment (ROI). 


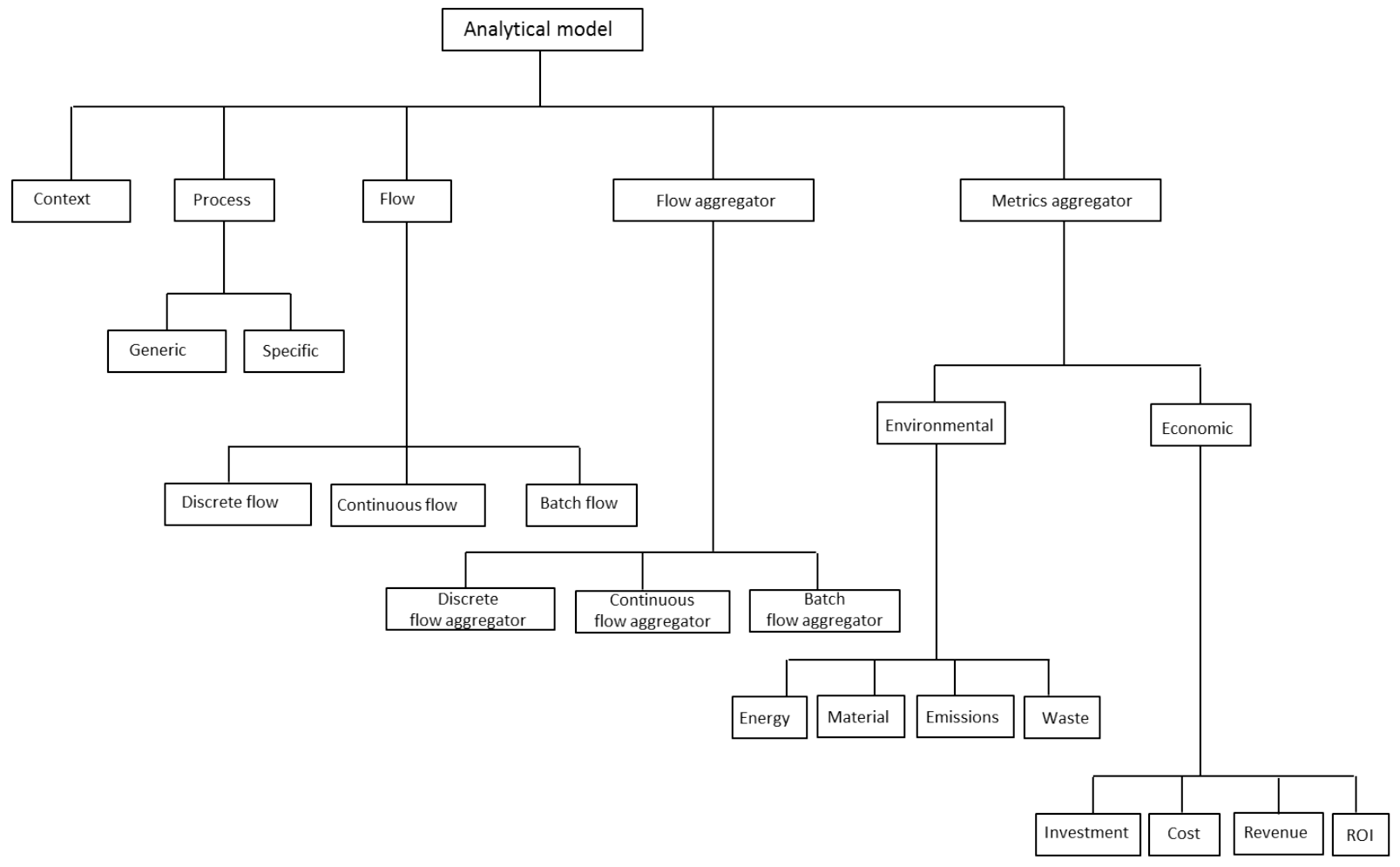

Figure 5 A hierarchical diagram for the process description and sustainability metrics model templates

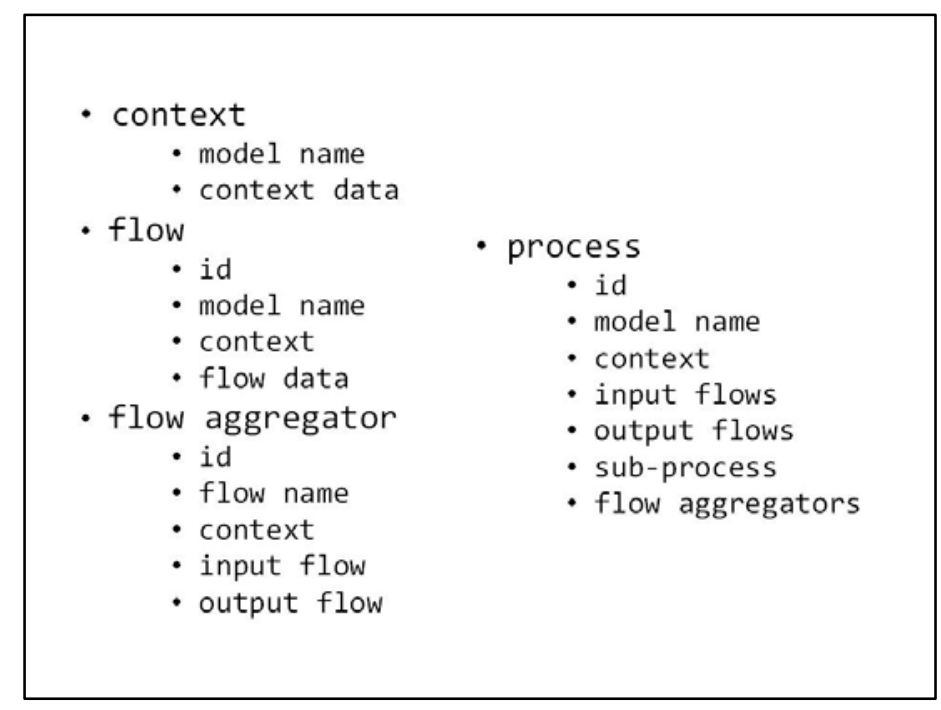

Figure 6 SPAF process description model components

SPAF libraries collect model components of both generic and specific models. Metrics model components can also be stored in a library. Figure 7 shows examples of generic model components in a SPAF library, e.g., process model components such as "baseSeqTransform" and "baseProcessComposer", and flow and 
flow aggregator models for discrete and continuous flows, and metrics models for environmental and economic indicators. Figure 8 lists examples of specific model components in the library; these models were developed for the two-product-manufacturing example. It includes specific process models for "machine A," "machine B," "machine C," “assembly A," “assembly B," and composite model "twoProdManuf" for the overall process. The model components in the SPAF library provide reusable building blocks and can be used as templates for a family of manufacturing processes; each model or template can be reused with some adjustment for different cases within the family. New models can be added to the SPAF library. Moreover, the existing models within the library can be executed with new data so that different companies that have the same problems could use the models by inputting their data to seek company-specific decision guidance. Using model components in the library, modelers can create SPAF models and queries for their operations more effectively and efficiently.

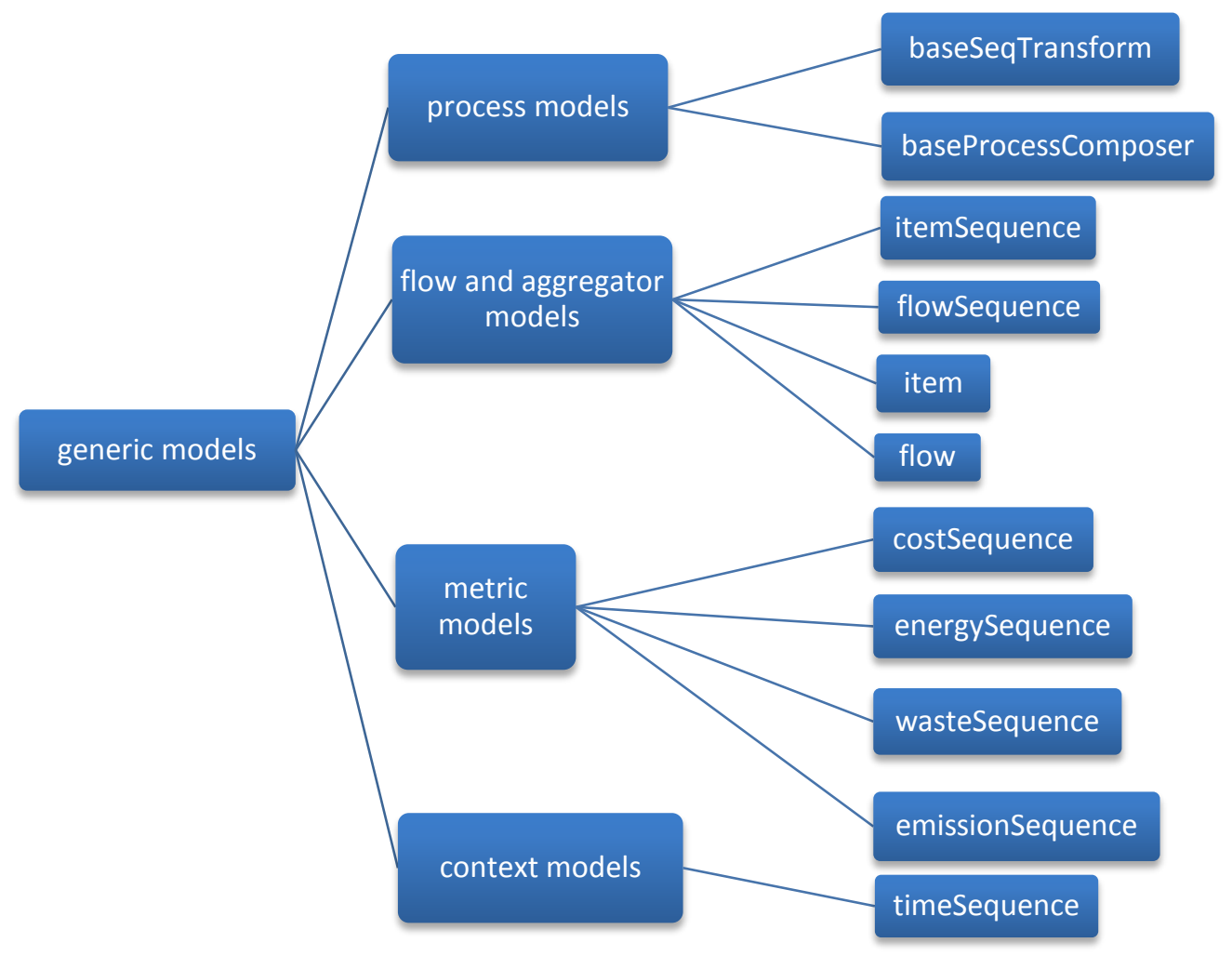

Figure 7 SPAF component library: example of generic models 


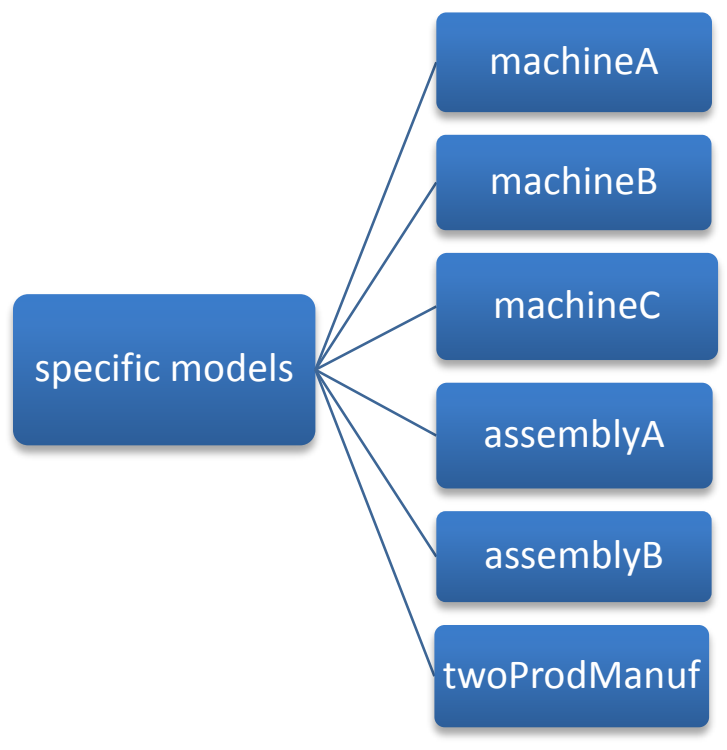

Figure 8 SPAF component library: examples of specific models

\subsection{Potential users of the SPAF}

Most people within the factory floor do not have the expertise to formulate and solve optimization problems using Mathematical Programming (MP) or Constraint Programming (CP). The SPAF makes process analysis modeling more intuitive and straight forward for domain users such as manufacturing engineers. Figure 9 shows a high-level use-case model for a typical implementation of a decision guidance management system using SPAF. There are three types of potential users, each with different roles in using and/or maintaining the system.

- SPAF analyst: The analyst is a primary user of the system. S/he may be an engineer who is in charge of defining case scenarios, setting up analysis model objectives together with decision makers, and identifying sustainability metrics, constraints, and controls. S/he will collect data from the factory floor and represent process structure, flow, and metrics using SPAF.

- Decision maker: The decision maker is the end user of the system. S/he identifies the SM objectives/goals and provides model requirements. The decision maker will query the knowledge base, ask what-if analysis questions, and/or make optimization requests with applied constraints and control data for a specific problem. S/he may also compose SPAF models simply using model components in the SPAF library.

- $\quad$ SPAF knowledge-base administrator: The knowledge-base administrator serves as a system administrator who is responsible for updating system data, creating reusable knowledge artifacts, helping the analyst develop new applications, maintaining the SPAF model library, modifying/improving the system design, and maintaining/enhancing the system. 


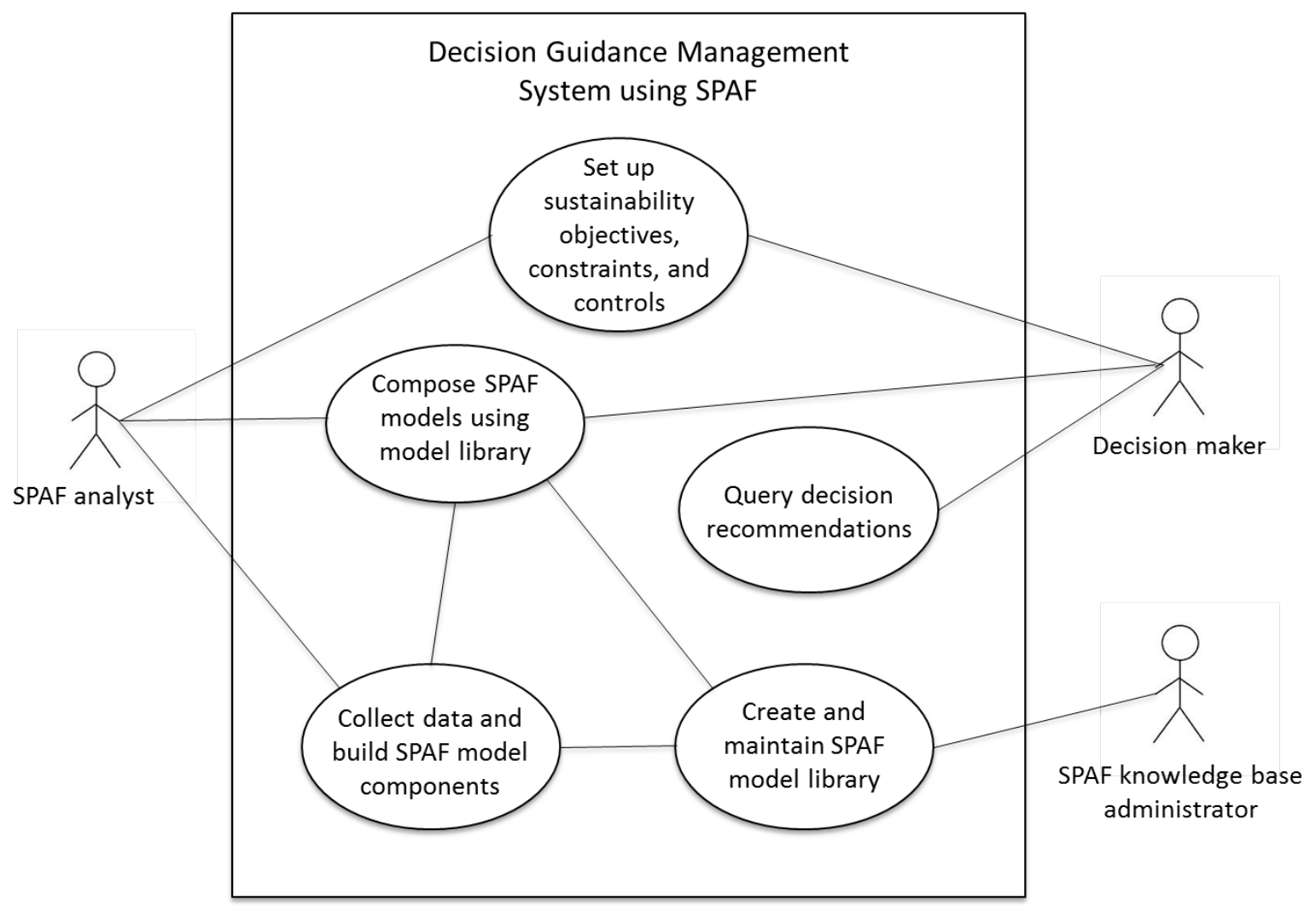

Figure 9 Use case for implementation of decision guidance management system using the SPAF

\section{ILLUSTRATIVE EXAMPLE USING SPAF}

In this section, the two-product-manufacturing example introduced in Section 3.2 is modeled using SPAF and discussed in details. Figure 10 to Figure 20 show the detailed SPAF process models and possible queries. First, assuming the SPAF model, twoProductsManuf (), is developed and all data are provided, a what-if analysis query requires only four statements (Figure 10). The first two statements include the data models for product demand data indicating quantities for each final product (i.e., Product 1 and Product 2) and production plan data that describes the numbers of components should be produced by each machining or assembly process. The third statement includes the SPAF model for the two-productmanufcturing process and finally a constratint statement that indicates the total amount of $\mathrm{CO}_{2}$ generated by this manufacturing scenario should be less than or equal to 50 metric tons. Since all data required for the query are available, the query is actually a deterministic computational model that calculates the total cost within the limit of total $\mathrm{CO}_{2}$ less than 50 metric tons. The query provides answers for both total cost and total $\mathrm{CO}_{2}$.
include data productDemand();
include data productionPlanData();
include process twoProductsManuf ();
twoProductsManuf.totalCO2 $\leq 50$;

Figure $10 \mathrm{~A}$ what-if analysis query for the two-product-manufacturing process 
In a case where the production plan data are not provided, an optimal production plan with minimal total cost needs to be determined, given the same $\mathrm{CO}_{2}$ limitation as a constraint. The same SPAF model, twoProductsManuf (), is used as shown in Figure 11, but the production plan data (as shown in Figure 10) is not provided and an optimizaiton statement is added to "minimize" the total cost. There are still only four lines of code; however, since the production plan data are unknown, all data previously provided explicitly in the production plan data model become decision variables that need to be instantiated to satisfy all the constraints. Now it is no longer a deterministic computational model. It actually describes a set of non-deterministic computational paths, each corresponding to an instantiation of set of values for the decision variables. Some of the non-deterministic computation paths are "feasible," i.e., they satisfy all of the constraints (the total $\mathrm{CO}_{2}$ constraints as well as the internal constraints) while others are not feasible. The semantics of the optimizaion query in Figure 11 is to find a non-deterministic optimizaiton path that leads to the minimal total cost among all feasible computation paths. The query results inlcude not only both total cost and total $\mathrm{CO}_{2}$ but also the optimal production plan configuation (i.e., the optimal number of components being produced by each machining or assembly process).

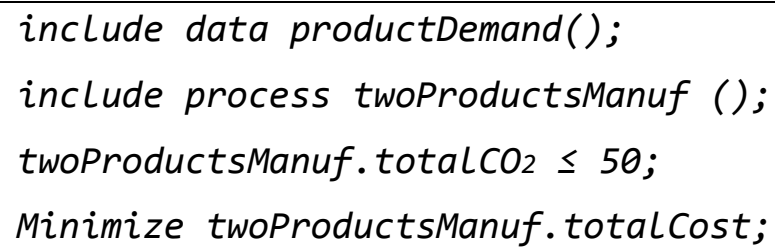

\section{Figure 11 Optimization query for the two-product-manufacturing process}

As stated above and shown in Figure 10 and Figure 11, the same SPAF model, twoProductsManuf (), can be used for different kind of queries, such as what-if analysis (in Figure 10) and decision optimization (in figure 11). These query examples demonstrate that SPAF provides a unified modeling capability. The queries against SPAF models are simple and straightforward.

Figure 12 shows the model for the "context" component, its model name is "timeSequence." "timeSequence" is declared as a set of string "day," which is a tuple consisting of three fields "day, month, and year" of type integer. The three dots "..." expresses the missing data that need to be instantiated as a constant, or an expression before the data is used.

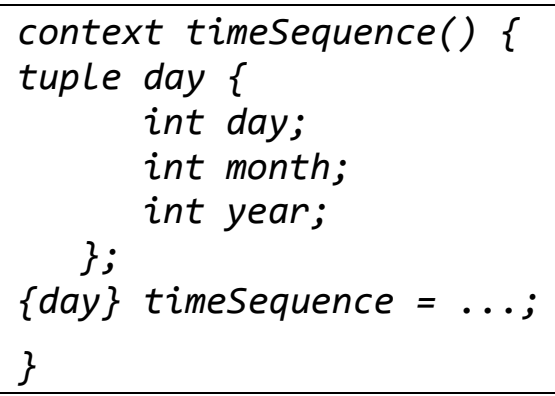

Figure 12 Context model for the two-product-manufacturing example

Figure 13 shows the model for the "flow" component, the model name is "itemSequence." The "Id"parameter of "itemSequence" will be replaced by the value of a parameter in an include statement. An include statement calls another model. It is similar to a subroutine call. The context model itemSequence () is included using a include statement. A one-dimensional array "Id.qty" is an integer 
array. "Id.qty" is indexed by the finite set of tuples defined by the "timeSequence" variable from the context model. The elements of the array represent quantities of the flow in that day.

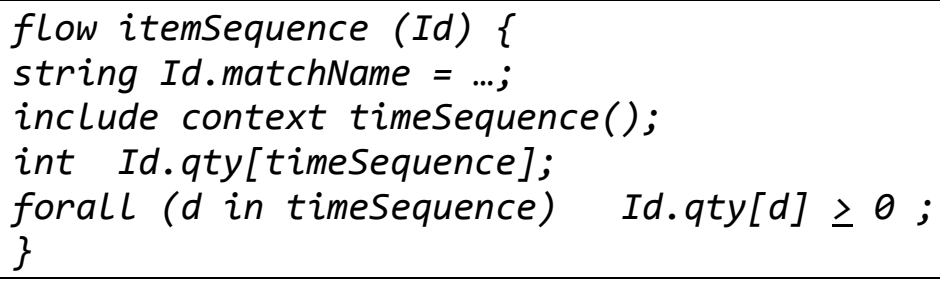

Figure 13 Flow model for the two-product-manufacturing example

Figure 14 shows a model for the "flow aggregator" component, its model name is "itemSeqAggr." As described earlier, "Id" is a parameter whose value will be provided by an include statement. In the first statement of this model, the context model itemSequence () is included. Next, a variable "Id.flowType" is declared as a string "itemSequence." "Id.inputFlows" and "Id.outputFlows" are declared as a set of strings and will be instantiated separately. "Id.flows," the union of "Id.inputFlows" and "Id.outputFlows," is also a set of strings. For every flow in "Id.flows," its quantity for the day in "timeSequence" is an integer. The forall statement defines a constraint for each day in "timeSequence," it indicates that the total number of the "inputFlows" for a day must equal the total number of the "outputFlows."

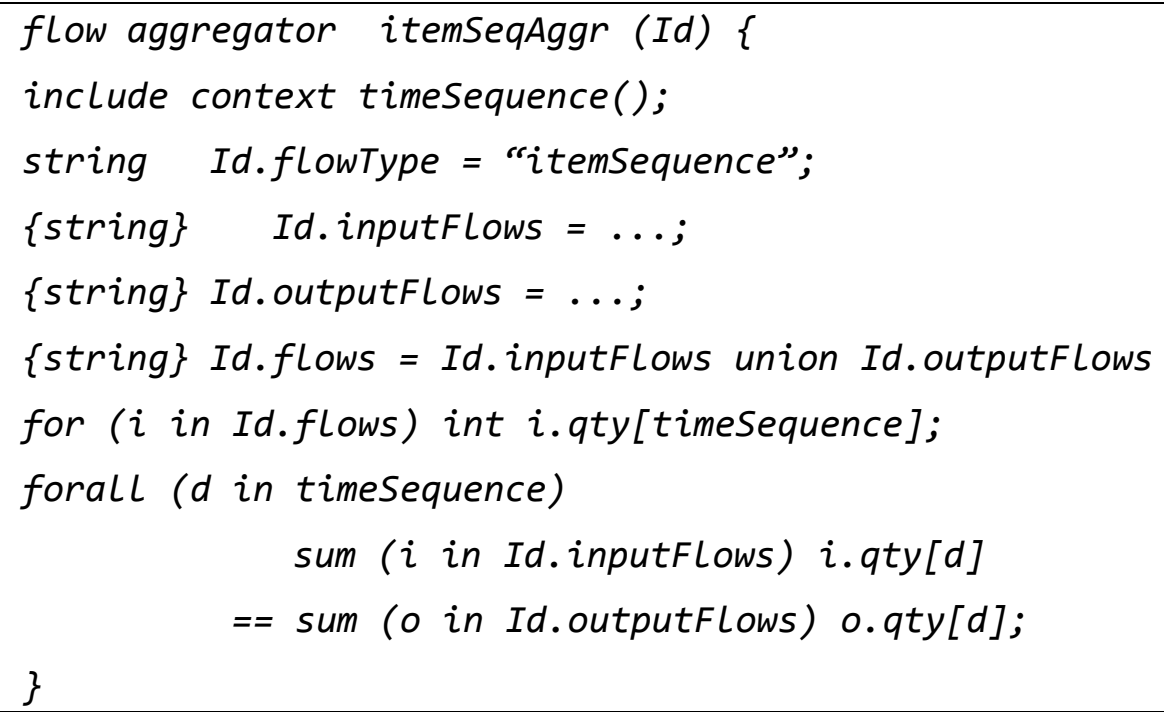

Figure 14 Flow aggregator model for the two-product-manufacturing example

Figure 15 shows a model of a generic atomic process, which is an end process in which there is no subprocess, e.g., Machine A. The model name is "baseSeqTransform." Id is provided when it is called. For every output flow, the flow model is being included with a parameter of the flow name. Two arrays of 
floats are declared for both "Id.costPerUnit" and "Id.CO $\mathrm{CO}_{2}$ PerUnit"; their index set is the set of output flows for this atomic process. A two-dimensional array of integer "Id.inputPerOutput" represents the number of input flows required for each output flow. For each production day, the cost of the atomic process is computed as the unit cost of each output flow times the number of output flows produced in that day; the $\mathrm{CO}_{2}$ emission is computed as the unit $\mathrm{CO}_{2}$ emission from each output flow times the number of output flows produced in that day. A constraint is that the total number of input flows needed in that day must equal the number of output flows produced in the same day times the number of input flows required for each output flow.

Once the generic atomic process model component is developed, it can be saved and reused for generating specific atomic process model components.

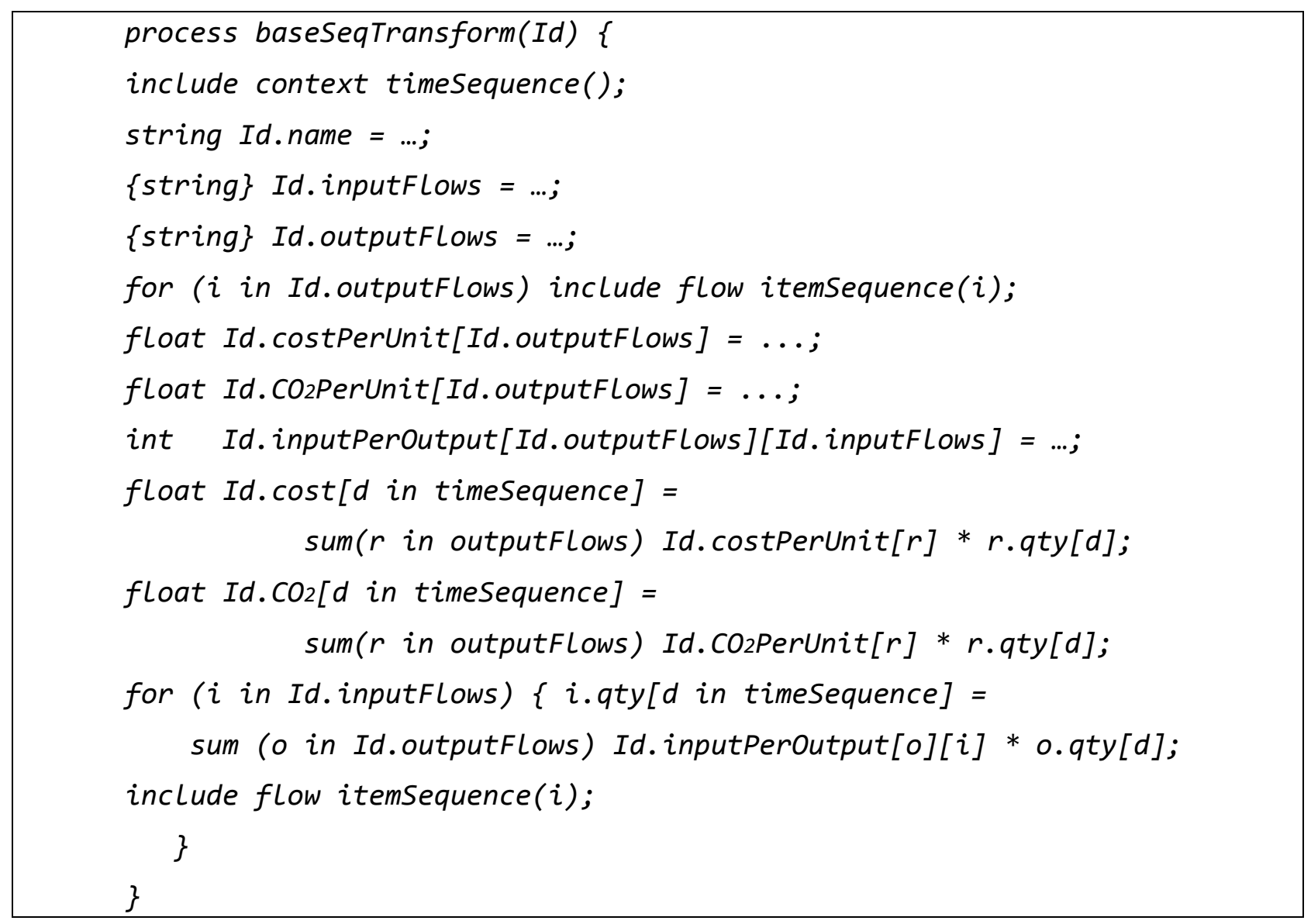

\section{Figure 15 An atomic process model for the two-product-manufacturing example}

Figure 16 shows a specific atomic process model for Machine A. Model name is "machine." It starts with the instantiation of the declarations. "Id.name" is given as "machine." "Id.inputFlows" is a set of two strings, "part1toMaA" and "part2toMaA." Input flows of "part1toMaA" and "part2toMaA" are given names "part1" and "part2" respectively. "Id.outputFlows" is a set of two strings "comp1fromMaA" and "comp2fromMaA." Output flows, "comp1fromMaA" and "comp2fromMaA," are given names of "comp1" and "comp2" respectively. Two float type arrays for "Id.costPerUnit" and "Id.CO $\mathrm{C}_{2}$ PerUnit" are both given in a pair (index, value) of elements as ["comp1fromMaA": 35.0, "comp2fromMaA": 65.0] and ["comp1fromMaA": 0.05, "comp2fromMaA": 0.02] respectively. The two-dimensional array 
"Id.inputPerOutput" is instantiated as a pair of ["comp1fromMaA": ["part1toMaA": 1,"part2toMaA": 1], "comp2fromMaA": ["part1toMaA": 1,"part2toMaA": 3]] The last step is to include the generic model "baseSeqTransform." "machineA" is the parameter.

Other atomic processes in the two-product-manufacturing example including Machine $\mathrm{B}$, Machine C, Assembly A, and Assembly B are similar to the process model of Machine A.

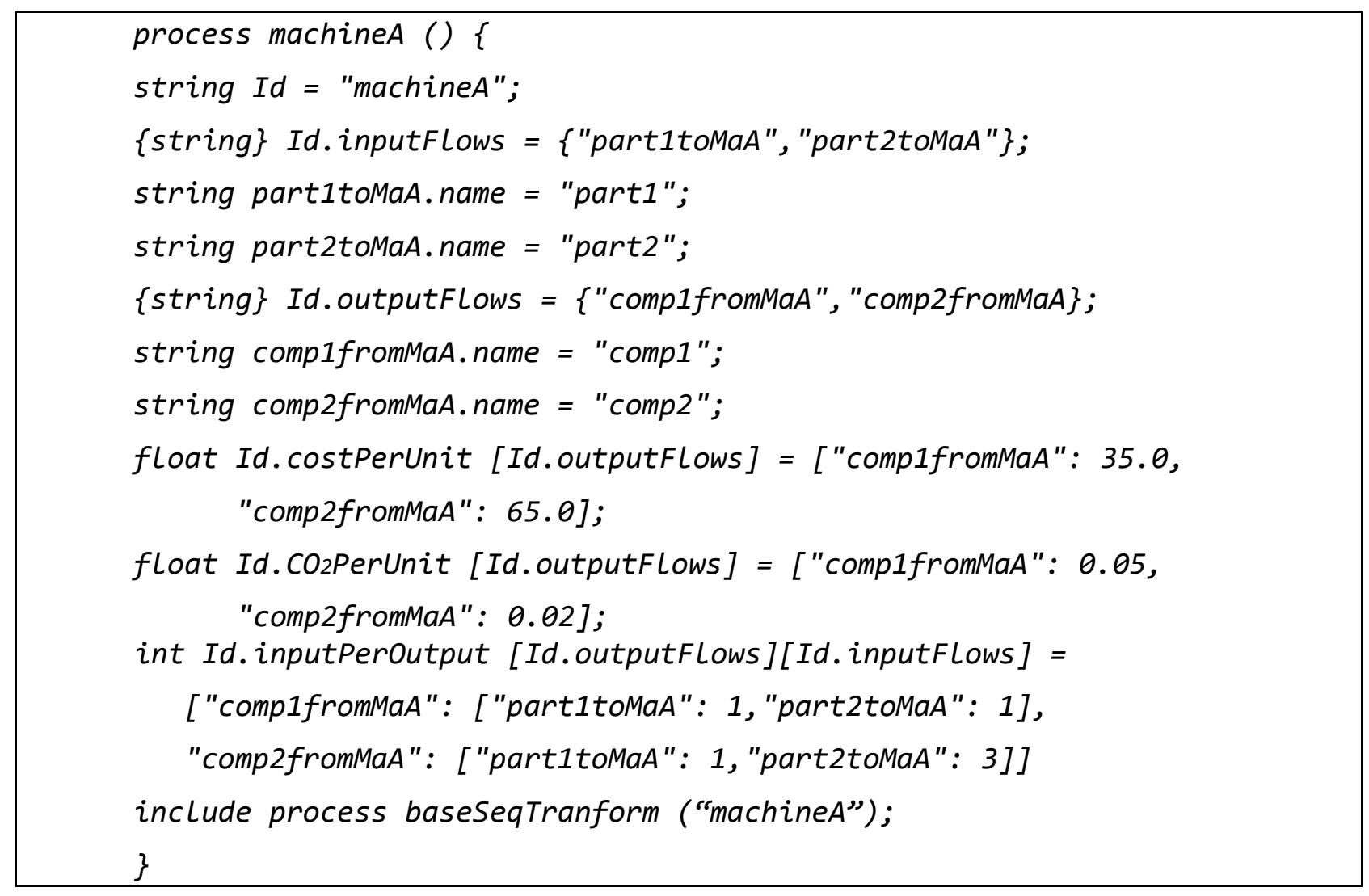

Figure 16 Atomic process model for Machine A

Depicted in Figure 17 is a generic process composer model, which includes all flow models and all subprocesses models, and formulates the flow aggregator models automatically instead of being given explicitly. Again, three dots indicate that the input and output flows, and sub-processes need to be instantiated before this generic model is called. For every flow, the model needs to be included and its model name, "matchName," and aggregator name need to be defined before this generic model is called. "flowsToAggregators," a set of strings, are the union of input flows to the composite process and output flows from all sub-processes. "flowsFromAggregators," another set of strings, are the union of all input flows to all sub-processes. All aggregator flow names are in the set of strings that include all "matchName" of the flows. For every flow "matchName," if the name of input flow is in the "flowsToAggregators" and the name of the output flow is in the "flowsFromAggregators," then include the flow aggregator model with the flow's "matchName” as a parameter.

process processComposer(id) \{

\{string\} Id. inputFlows $=\ldots$;

\{string Id.outputFlows $=\ldots$; 


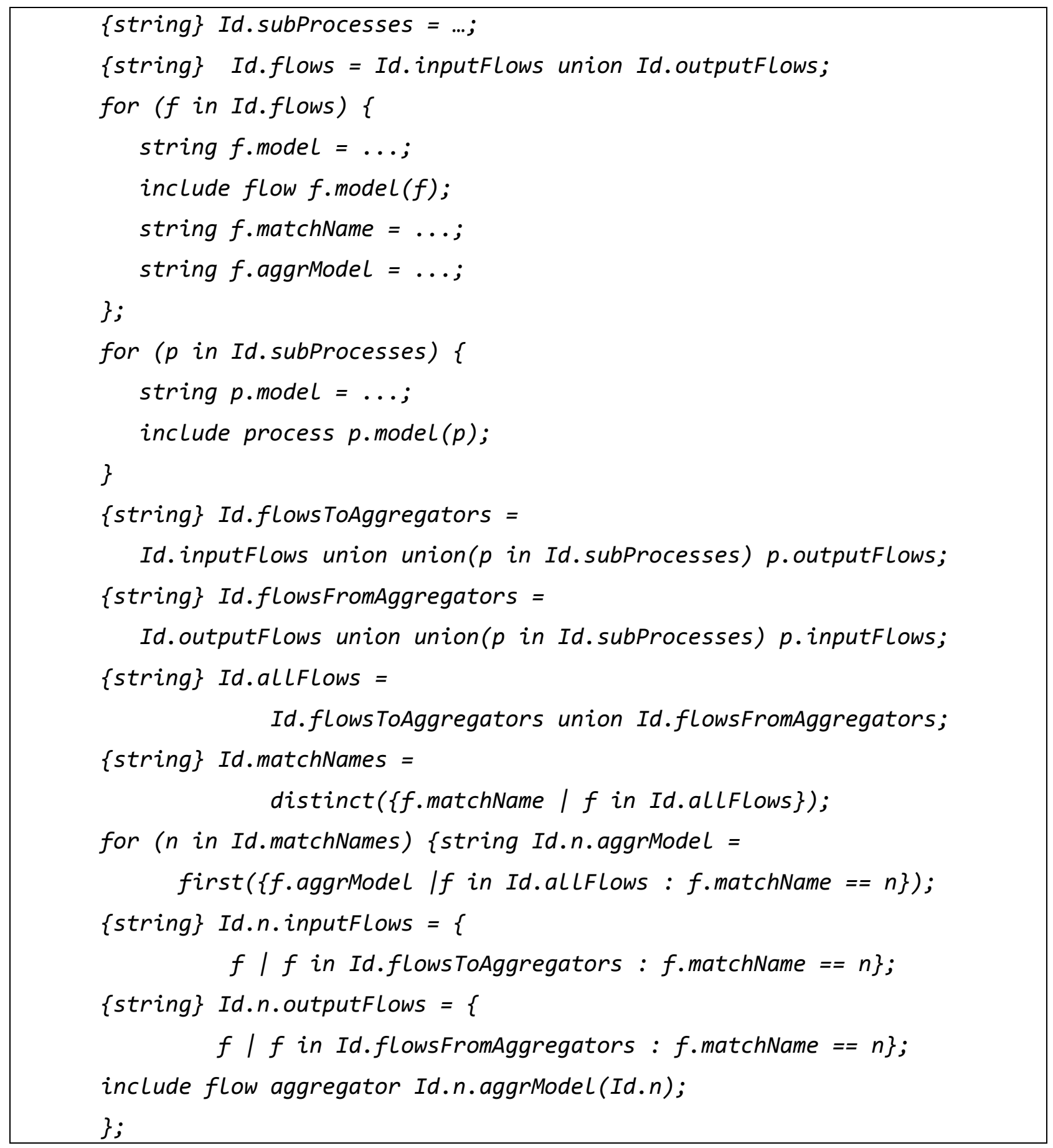

\section{Figure 17 Generic composite process model}

Figure 18 shows the metrics aggregator models that compute daily total cost and $\mathrm{CO}_{2}$. The daily total cost and $\mathrm{CO}_{2}$ are the sum of cost and $\mathrm{CO}_{2}$ for all sub-processes.

metric aggregator costSequence(Id) \{

include context timesequence (); 


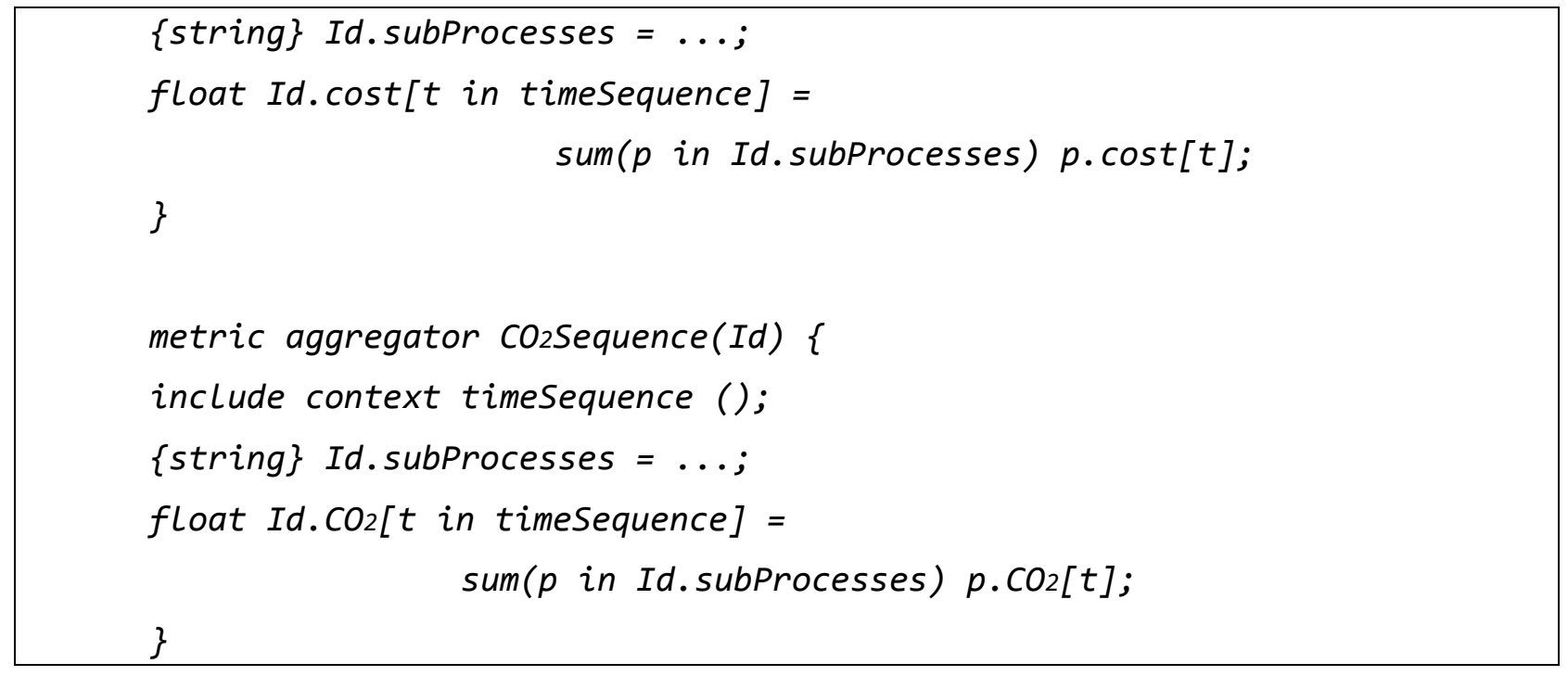

\section{Figure 18 Metric aggregator model}

Figure 19 shows the composite process model. Model Id is "twoProductsManuf." It includes the context model itemSequence (). "Id.inputFlows" is given as a set of two strings \{"part1in", "part2in"\}. "Id.outputFlows" is given as a set of two strings of \{"product1", "product2"\}. "matchNames" are also given. "Id.subProcessess" is instantiated as a set of five strings of \{ "machine," "machineB", "machine," "assembly," "assemblyB" \}. The generic process model processComposer is called to include all atomic sub-processes models defined previously. Float type of data for extra facility cost and $\mathrm{CO}_{2}$ per day (\$1750 and 0.3 metric tons) are provided. The metric aggregator models, costSequence (Id) and $\mathrm{CO}_{2}$ Sequence(Id), are included. Total cost for each day is the extra facility cost plus daily cost for all subprocesses. Total $\mathrm{CO}_{2}$ for each day is the extra facility $\mathrm{CO}_{2}$ plus total sub-processes $\mathrm{CO}_{2}$.

An alternative modeling method is to explicitly instantiate all flows and flow aggregators, e.g., inputs and outputs of Part1, Part2, Comp1, Comp2, and Comp3 are all specified as sets of strings. Then every flow aggregator is included with its name as a parameter.

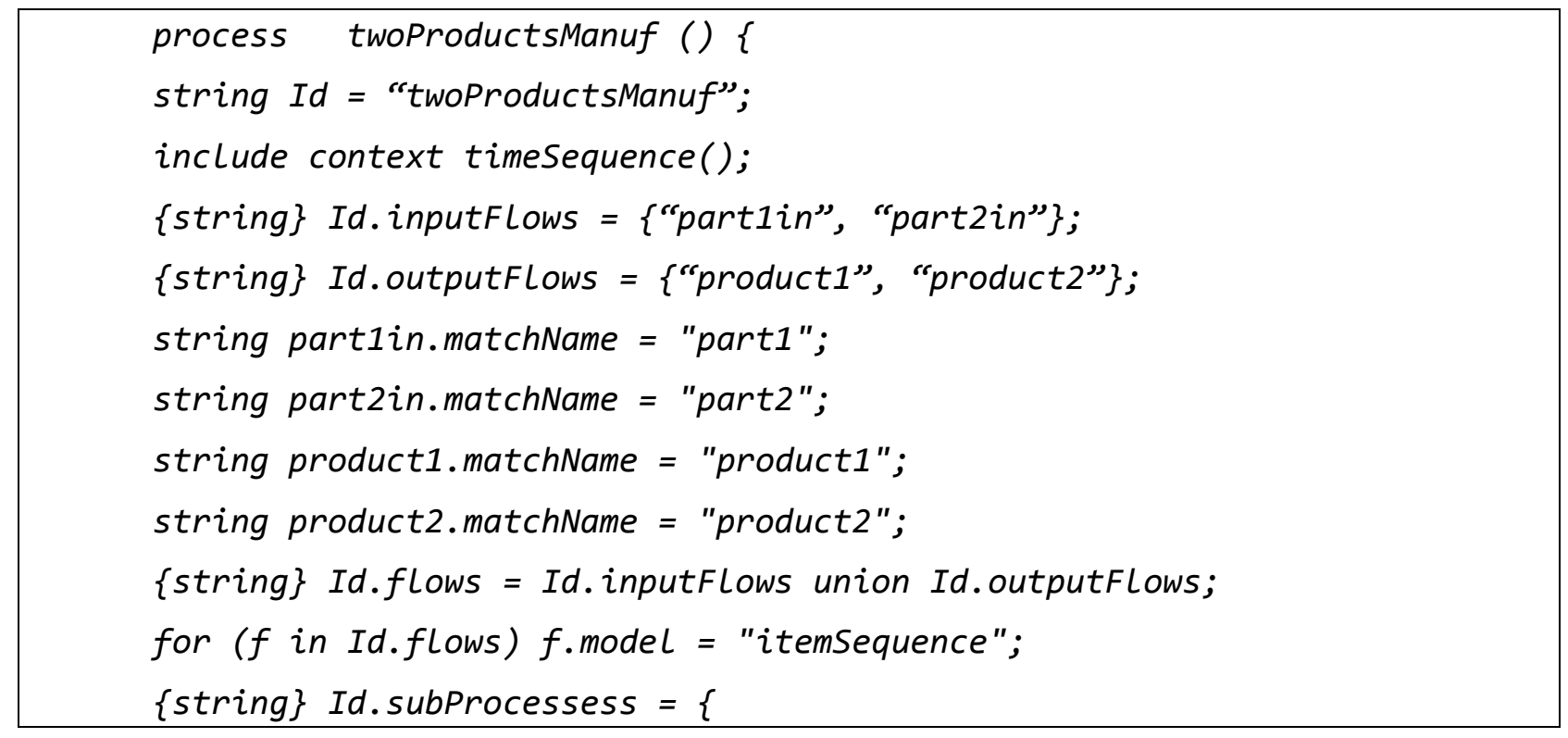




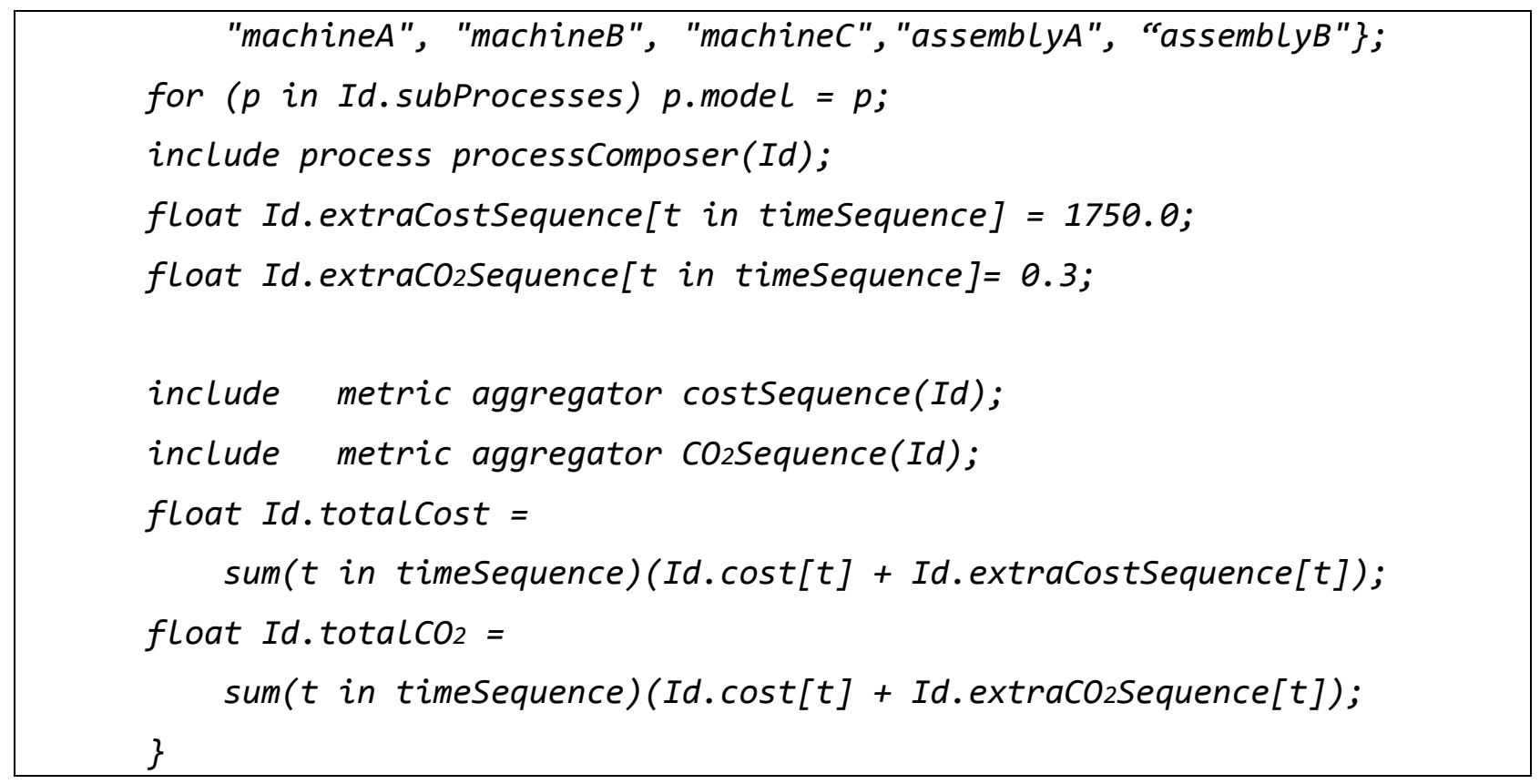

Figure 19 Composite process model for the two-product-manufacturing process

After we explained all the SPAF model components for the example, we need to examine the data required by the queries in Figure 10 and Figure 11. A context data sequence is shown in Figure 20. Its product demand data model is listed in Figure 21, in which the quantities of the two products are given for each production day. For example, $[<4,9,2012>$ : 6] in the first line means demand for Product 1 on September $4^{\text {th }}$, 2012 is 6.

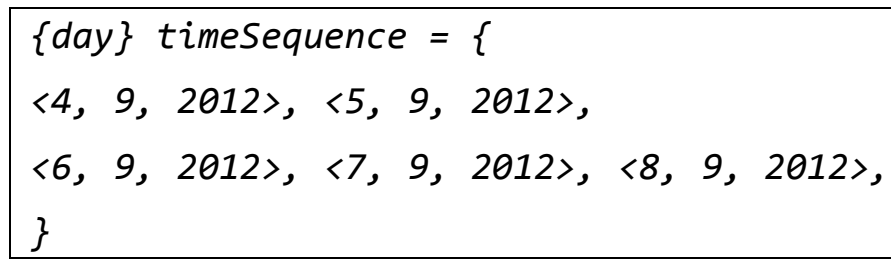

Figure 20 A context data sequence for the two-product-manufacturing process

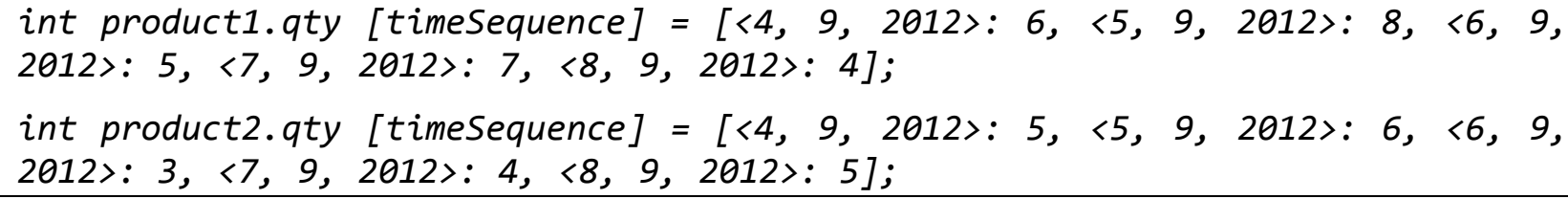

Figure 21 Product demand data model for product 1 and product 2

A what-if scenario for the example is described as follows: if the process engineer uses a predefined production plan, i.e., all the data such as numbers of part 1 and part 2, numbers of components flows into 
and out of Machine A, Machine B, Machine C, and number of components flows into Assembly A, and Assembly B each day are fixed. This means that all the data needed in the SPAF model are explicitly provided and can be used to computer metrics using formulas. The four lines of what-if query (as shown in Figure 10) can be expanded as in Figure 22 while the constraint keeps the same as before.

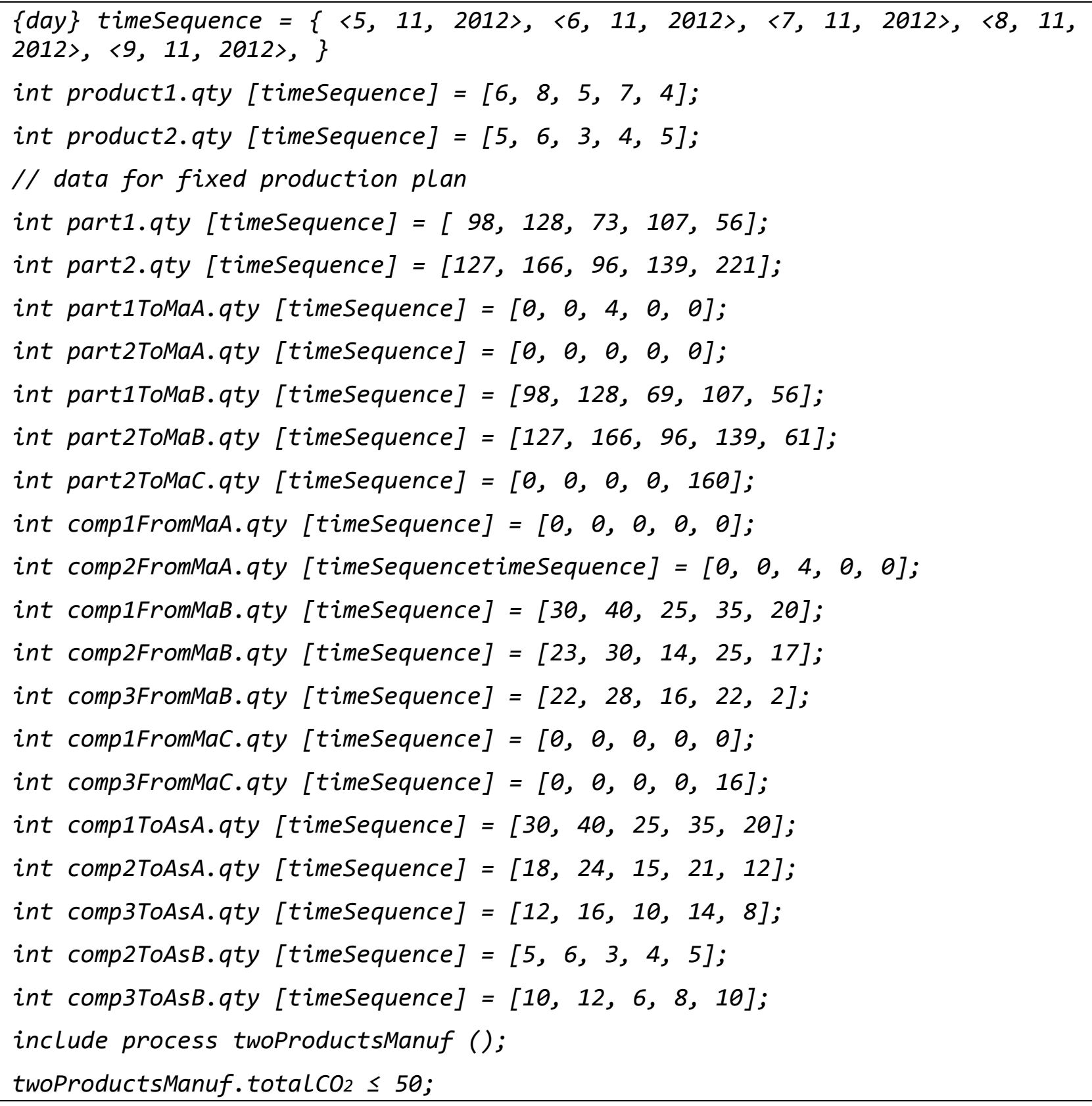

Figure 22 What-if query for the two-product-manufacturing example

This is a deterministic computational model, however, since there is a constraint statement in the query and there are also other data integrity constraints within the models, the answers have to satisfy all the constraints. The results of the what-if scenario are: the total cost is $\$ 30000$ with a total of 35.11 metric tons of $\mathrm{CO}_{2}$. Note that changes in any input data will result in a different set of solutions. 
For the optimization query listed in Figure 11, input data such as weekly production schedule and customers' demand for Product 1 and Product 2 are provided. The sustainability goal is to determine an optimal production plan that minimizes the total cost within a $\mathrm{CO}_{2}$ bound of 50 metric tons. The optimization model performs multiple non-deterministic computations, each instantiates decision variables (quantities of flows in each configuration) using values that satisfy all the constraints. Among those sets of configurations that satisfy all the constraints, the system will automatically find a configuration (i.e., a production plan) that minimizes the total cost. Figure 23 shows the optimization result screen of an implementation using IBM ILOG CPLEX. The optimal production plan for the scheduled five days is derived. The optimization results show that the minimal total cost is \$28 023 with total 36.72 metric tons of $\mathrm{CO}_{2}$. The results also indicate that due to the higher operation cost of the Machine B, it is not recommended to use Machine B to produce any of the components, i.e., Comp1, Comp2, and Comp3. Note that changes in any of the input data and constraints will also affect the values of decision variables and decision expressions.

\begin{tabular}{|c|c|c|}
\hline \multicolumn{2}{|l|}{ Solution with objective 28,023} & \multirow{2}{*}{$\vec{T}$} \\
\hline Name & Value & \\
\hline${ }^{f}$ machineB_outputFlows & \{"comp1fromMaB" "comp2fromMaB" "comp3fromMaB"\} & \\
\hline Fi machineC_CO2PerUnit & {$[0.150 .06]$} & \\
\hline Fio machineC_costPerUnit & [45 58] & \\
\hline${ }^{A}$ machineC_inputFlows & $\{"$ part2toMaC"\} & \\
\hline Il ${ }^{10}$ machineC_inputPerOutput & {$[[5][10]]$} & \\
\hline$f^{A}$ machineC_outputFlows & $\{"$ comp1fromMaC" "comp3fromMaC"\} & \\
\hline Ilo prodifromAsA_qty & {$[68574]$} & \\
\hline Iil prod2fromAsB_qty & [5 636345$]$ & \\
\hline$\langle$ productionSequence & $\{<492012\rangle<592012\rangle<692012\rangle<792012\rangle<892012\rangle\}$ & \\
\hline \multicolumn{3}{|l|}{ Decision variables $(9)$} \\
\hline 望 comp1fromMaA_qty & 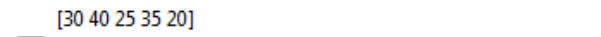 & \\
\hline Fil comp1fromMaB_qty & {$\left[\begin{array}{llllllll}0 & 0 & 0 & 0 & 0\end{array}\right]$} & \\
\hline 望 complfromMaC_qty & {$\left[\begin{array}{lllll}0 & 0 & 0 & 0 & 0\end{array}\right]$} & \\
\hline 笛 comp2fromMaA_qty & {$\left[\begin{array}{llllllllll}23 & 30 & 18 & 25 & 17\end{array}\right]$} & \\
\hline Fil comp2fromMaB_qty & 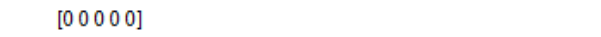 & \\
\hline Fip comp3fromMaB_qty & {$\left[\begin{array}{lllll}0 & 0 & 0 & 0 & 0\end{array}\right]$} & \\
\hline Fip comp3fromMaC_qty & {$\left[\begin{array}{lllllllllll}22 & 28 & 16 & 22 & 18\end{array}\right]$} & \\
\hline Fin partlin_qty & [53 704360 37] & \\
\hline$=10$ part2in_qty & {$\left[\begin{array}{llllll}220 & 280 & 160 & 220 & 180\end{array}\right]$} & \\
\hline \multicolumn{3}{|l|}{ Decision expressions (24) } \\
\hline FisemblyA_CO2 & {$\left[\begin{array}{llllllll}3 & 4 & 2.5 & 3.5 & 2\end{array}\right]$} & \\
\hline Fi AssemblyA_cost & {$\left[\begin{array}{llllllllll}60 & 80 & 50 & 70 & 40\end{array}\right]$} & \\
\hline Fo AssemblyB_CO2 & {$[11.20 .60 .81]$} & \\
\hline$\vec{P}$ AssemblyB_cost & 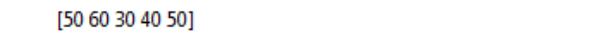 & \\
\hline $\overrightarrow{\mathrm{F}} \mathrm{CO} 2$ & [7.48 9.68 5.87 8.07 5.62] & \\
\hline =10 comp1toAsA_qty & 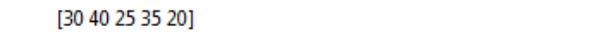 & \\
\hline =10 comp2toAsA_qty & {$\left[\begin{array}{lllllll}18 & 24 & 15 & 21 & 12\end{array}\right]$} & \\
\hline Fip comp2toAsB_qty & 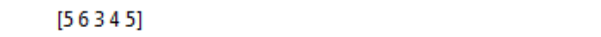 & \\
\hline =10 comp3toAsA_qty & {$[121610148]$} & $\equiv$ \\
\hline Ifor comp3toAsB_qty & {$[10126810]$} & \\
\hline Fi cost & [5681 6864480359864689$]$ & \\
\hline $\overrightarrow{\mathrm{F}}$ machineA_CO2 & 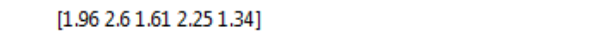 & \\
\hline Pi machineA_cost & {$\left[\begin{array}{llllll}2545 & 3350 & 2045 & 2850 & 1805\end{array}\right]$} & \\
\hline 承 machineB_CO2 & {$\left[\begin{array}{lllllllllllll}0 & 0 & 0 & 0 & 0\end{array}\right]$} & \\
\hline Fi: machineB_cost & {$\left[\begin{array}{lllll}0 & 0 & 0 & 0 & 0\end{array}\right]$} & \\
\hline $\overrightarrow{\mathrm{F}} \mathrm{e}$ machineC_CO2 & {$[1.321 .680 .961 .321 .08]$} & \\
\hline Fi machineC_cost & [1276 162492812761044$]$ & \\
\hline Fip partitoMaA_qty & [53 70436037$]$ & \\
\hline Fie part1toMaB_qty & {$\left[\begin{array}{llllll}0 & 0 & 0 & 0 & 0\end{array}\right]$} & \\
\hline$=10$ part2toMaA_qty & {$\left[\begin{array}{llllll}0 & 0 & 0 & 0 & 0\end{array}\right]$} & \\
\hline =10 part2toMaB_qty & {$\left[\begin{array}{lllllllllllllll}0 & 0 & 0 & 0 & 0\end{array}\right]$} & \\
\hline =10 part2toMaC_qty & {$\left[\begin{array}{llllll}220 & 280 & 160 & 220 & 180\end{array}\right]$} & \\
\hline .0 totalCO2 & 36.72 & \\
\hline .0 totalCost & 28023 & T \\
\hline
\end{tabular}

Figure 23 Optimal solution screen of two-product-manufacturing example 


\section{CONCLUSION AND FUTURE WORK}

This paper proposed a NIST-developed Sustainable Process Analytics Formalism that allows manufacturers to: (1) formally represent sustainable process structure, flow, process data, control variables, and process analytical model of sustainability metrics and constraints for quantitative sustainability analysis; and (2) analyze and make decisions on improvement alternatives with modeling and optimization tools. The formalism provides platform-independent process-knowledge description and supports what-if analysis and decision optimization for decision makers. The use of the SPAF formalism is illustrated through a two-product manufacturing process example. The SPAF syntax, formal semantics, and query computation algorithm are presented in the appendix.

The formalism will be deployed to industry through case studies and contributions to standard development efforts. When implemented for real manufacturing applications, the formalism will help manufacturers quantify their sustainability efforts for improvement of energy and material efficiency, lower emissions, and save cost.

Future work includes (1) examining diverse manufacturing processes to identify extra process analytical needs; (2) supporting taxonomies, and metrics from unit manufacturing, assembly processes, and production planning; (3) supporting smart manufacturing by enhancing the SPAF; (4) developing translators that automatically translate SPAF to formal optimization/simulation models, which can then be solved by commercial optimization tools; (5) developing graphical representation of SPAF based on modeling language such as UML, SysML, or BPMN; (6) performing industrial case studies to evaluate and validate the formalism and the capabilities; and (7) standardizing the SPAF.

\section{DISCLAIMER}

No approval or endorsement of any commercial product by the National Institute of Standards and Technology is intended or implied. Certain commercial software systems are identified in this paper to facilitate understanding. Such identification does not imply that these software systems are necessarily the best available for the purpose.

\section{REFERENCES}

AMPL. (2011). “A Modeling Language for Mathematical Programming.” Available via $<$ http://www.ampl.com/> [accessed Jan. 2012].

Berglund, J. K., Michaloski, J. L., Leong, S. K., Shao, G., Riddick, F. H., Arinez, J., et al. (2011). Energy Efficiency Analysis for a Casting Production System. Proceedings of the 2011 Winter Simulation Conference, (pp. 1060-1071).

Brodsky, A., and Nash, H. (2005). CoJava: a unified language for simulation and optimization. The Conference on Object Oriented Programming Systems Languages and Applications, (pp. 194 195).

Brodsky, A., and Wang, S. X. (2008). Decision-Guidance Management System (DGMS): Seamless Integration of Data Acquisition, Leaning, Prediction, and Optimization. The 41st Annual Hawaii International Conference on System Sciences (HICSS 2008), (pp. 71-81). Hawaii.

Chieh, C. (2010). Six Sigma Basics: DMAIC Like Normal Problem Solving. Available via < http://www.isixsigma.com/new-to-six-sigma/dmaic/six-sigma-basics-dmaic-normal-problemsolving/> [accessed August. 15, 2013].

CPLEX. (2011). Available via <http://en.wikipedia.org/wiki/CPLEX> [accessed August. 2013]. 
DOC. (2010). Sustainable Manufacturing Initiative and Public-private Dialogue. Available via $<$ http://www.trade.gov/competitiveness/sustainablemanufacturing/index.asp $>$ [accessed Jan. 15, 2012].Fujitsu. (2011). Fujitsu Offers Energy-Saving Green Infrastructure Solution. Available via <http://www.fujitsu.com/global/news/pr/archives/month/2007/20071210-02.html> [accessed March 2012].

GAMS. (2010). An Introduction to General Algebraic Modeling System (GAMS). Available via $<$ http://www.gams.com/> [accessed July 2013].

GM. (2010). Innovation: Environment. Available via

<http://www.gm.com/corporate/responsibility/environment/facilities/index.jsp> [accessed August 2012].

Feng, S. C. and Joung, C. B. (2009). An Overview of a Proposed Measurement Infrastructure for Sustainable Manufacturing. Proceedings of the 7th Global Conference on Sustainable Manufacturing.

IBM. (2012). Introducing IBM ILOG CPLEX Optimization Studio V12.2. Available via $<$ http://pic.dhe.ibm.com/infocenter/cosinfoc/v12r2/index.jsp?topic=\%2Filog.odms.ide.help\%2FC ontent\%2FOptimization\%2FDocumentation\%2FOPL_Studio\%2F_pubskel\%2Fglobals\%2Feclips e_and_xplatform\%2Fps_opl307.html>. [accessed August 2013].

ISO 18629-1:2004. (2004). Industrial Automation Systems and Integration - Process Specification Language - Part 1: Overview and Basic Principles. Available via $<$ http://www.iso.org/iso/iso_catalogue/catalogue_tc/catalogue_detail.htm?csnumber=35431> [Access Sept. 2013].

ISO/IEC 9075-1:2011. (2011). Information technology - Database languages - SQL - Part 1: Framework (SQL/Framework). Available via $<$ http://www.iso.org/iso/iso_catalogue/catalogue_tc/catalogue_detail.htm?csnumber=53681> [Access Sept. 2013].NIST. (2010). Metrics, Standards, and Infrastructure for Sustainable Manufacturing workshop. Gaithersburg, MD: Available via <http://www.mel.nist.gov/msid/conferences/Agenda_SMW.htm > [access August 2013].

NIST SM. (2012). Sustainable Manufacturing Program. Available via <http://www.nist.gov/el/msid/lifecycle/sustainable_mfg.cfm> [accessed Sept. 2013].

National Research Council. 1999. Industrial Environmental Performance Metrics: Challenges and Opportunities. Washington, DC: The National Academies Press.

OECD. (2013). Sustainable Manufacturing Toolkit Prototype. Available via < http://www.oecd.org/innovation/green/toolkit/48661768.pdf [accessed Sept. 2013].

OMG. (2010). Business Process Model and Notation (BPMN). Available via <http://bpmnhandbook.com/01_specs/BPMN_20_spec.pdf> [accessed Sept. 2013].

OMG. (2012). OMG Systems Modeling Language. Available via <http://www.omgsysml.org/> [accessed Sept. 2013].

OPL.

http://pic.dhe.ibm.com/infocenter/cosinfoc/v12r2/index.jsp?topic=\%2Filog.odms.ide.help\%2FConten t\%2FOptimization\%2FDocumentation\%2FOPL_Studio\%2F_pubskel\%2Fglobals\%2Feclipse_and_xp latform\%2Fps_opl307.html. [accessed Sept. 2013].

Paju, M., Heilala, J., Hentula, M., Heikkila, A., Johansson, B., Leong, S., and Lyons K. (2010). Framework and Indicators for a Sustainable Manufacturing Mapping Methodology. Proceedings of 2010 Winter Simulation Conference.

Pegden, C, Sadowski, R, Shannon, R. (1995). Introduction to Simulation Using SIMAN. McGraw-Hill, Inc. New York, NY. Pineda-Henson, R., Culaba, A. B. (2002). Developing an Expert System for GP Implementation. Proceedings of the 2nd World Conference on Green Productivity. Available via

http://www.apotokyo.org/gp/manila_conf02/resource_papers/narrative/henson_experta4.pdf [accessed August, 2013]. 
Rockwell Automation. (2010). Taking Energy Management to a Higher Level. Available via < http://www.managingautomation.com/maonline/research/download/view/Taking_Energy_Manageme nt_to_a_Higher_Level_27756351>. [accessed August 2013].

Shao, G., Kibira, D., Brodsky, A., \& Egge, N. (2011). Decision Support for Sustainable Manufacturing using Decision Guidance Query Language. The International Journal of Sustainable Engineering,Volume 4, Issue 3 , 251-265.

SMLC. 2011. Implementing 21st Century Smart Manufacturing. https://smart-processmanufacturing.ucla.edu/about/news/Smart\%20Manufacturing\%206_24_11.pdf. [accessed August 2013].

Tamer G. (2011). "Methodological study on technology integration for sustainable manufacturing in the surface finishing industry." ETD Collection for Wayne State University. Paper AAI3469974. http://digitalcommons.wayne.edu/dissertations/AAI3469974

Tanzil, T. and Beloff, B. (2006). “Assessing impacts: Overview on sustainability indicators and metrics,” Environmental Quality Management, Volume 15, Issue 4, pp. 41-56.

Waller, A. (2012). Witness Simulation Software. Proceedings of the 2012 Winter Simulation Conference. 


\section{Appendix: Sustainable Process Analytics Formalism Syntax and Formal Semantics}

\section{A. SPAF model syntax}

SPAF adopts concepts and ideas from other languages and is based on the OPL data model and the basic OPL syntax of arithmetic and query expressions with minor modifications and extensions. The basic OPL data model, modeling concept, data type, and data structure are listed in (IBM, 2012).

Analytical sequence, Aseq, is a sequence $\left(s_{1}, \ldots, s_{n}\right)$ of analytical statements, $s_{i,}$

$1 \leq i \leq n$, in one of the forms:

1. $T_{i} \quad x_{i}=a_{i}$

2. $T_{i} \quad x_{i}=e_{i}$

3. $T_{i} x_{i}$

4. $T_{i} \quad x_{i}=\ldots$

5. $C_{i}$

6. include $M_{i}(J)$ or include $M_{i}()$

7. $\min x_{i}, \max x_{i}$, or sat

where:

- The statements $\min x_{i}, \max x_{i}$, or sat are only allowed as the last statement $\mathrm{s}_{\mathrm{n}}$

- $T_{i}$ is a type

- $x_{i}$ is a variable name, which may include a prefix identifier, e.g., Id.x.

- $a_{i}$ is a constant of type $T_{i}$

- $e_{i}$ is an expression returning type $T_{i}$

- “..." is a keyword in " $T_{i} x_{i}=\ldots$ ” to indicate that $x_{i}$ is to be instantiated with a constant before using it later in the sequence

- $C_{i}$ is a constraint

- $M_{i}$ is a unique name of an analytical model

- $J$ in $M_{i}(J)$ is a string identifier

The first four forms are declaration statements, within which the first two forms are assignment statements. $C_{i}$ is a constraint statement, include $M_{i}$ is an include statement, and $\min x_{i}$, $\max x_{i}$, or sat are optimization statements, i.e., minimization, maximization, and satisfiability. If the last statement $s_{n}$ of the analytical sequence $\left(s_{1}, \ldots, s_{n}\right)$ is $\min x_{i}$, $\max x_{i}$, or sat, then the $\left(s_{1}, \ldots, s_{n}\right)$ is an optimization analytical sequence; otherwise, we say that it is a non-optimization analytical sequence.

An analytical model is an expression of the form

$M(I d)\{$ Aseq $\}$ or $M()\{$ Aseq $\}$

where $M$ is a unique name of the model, $I d$ is an optional parameter, and Aseq is a non-optimization analytical sequence.

Let $P$ be a set of analytical models. We say that $P$ is closed under reference (or closed) with respect to an analytical sequence $A$ (or model $M$ ) if the following holds: If an $A$ has a statement of the form include $M^{\prime}(J)$, then $P$ must contain an analytical model $M^{\prime}$. We say that $P$ is closed under reference (or closed) if for every model $M$ in $P, P$ is closed with respect to $M$.

An analytical query is a pair $(A, P)$, where $A$ is an analytical sequence and $P$ is a model package closed with respect to $A$.

Let $(A, P)$ be an analytical query. The flattened sequence of $A$, denoted flat $(A, P)$, is an analytical sequence that results from $A$ by recursively replacing each include $M($ ) with the analytical sequence of 
the model $M(\mathrm{)}$, and replacing each include $M(J)$ statement with the analytical sequence of the model $M(I d)$ in $P$, in which every appearance of $I d$ is replaced with $J$.

We say that an analytical query $(A, P)$ has a conflict, if one of the following holds in flat $(A, P)=\left(S_{1}, \ldots, S i, \ldots, S_{j}, \ldots, S_{n}\right)$ :

- $\mathrm{S}_{j}$ is a declaration statement of the form $T_{i} x_{i}=a_{i}$ or $T_{i} \quad x_{i}=e_{i}$ and $\mathrm{S}_{i}$ is any declaration statement

- $S_{i}$ and $S_{j}$ are two declaration statements such that $\mathrm{x}_{i}=\mathrm{x}_{j}$ and $\mathrm{T}_{i} \neq \mathrm{T}_{j}$ (i.e., the same variable is declared twice with conflicting types)

Given a flat $\left(S_{1}, \ldots, S_{i}, \ldots, S_{n}\right)$ analytical sequence $A$ (i.e., without include statement), we say that variable $x_{i}$ is data-instantiated if:

- There is a statement $S_{i}$ of the form $T_{i} \quad x_{i}=a_{i}$, where $a_{i}$ is a constant or, recursively

- There is a statement $S_{i}$ of the form $T_{i} \quad x_{i}=e_{i}$, such that all variables y in $e_{i}$ are instantiated in the prefix sequence $\left(\mathrm{S}_{1}, \ldots, \mathrm{S}_{\mathrm{i}-1}\right)$

We say that a flat analytical sequence $A$ is data instantiated if every variable $\mathrm{x}$ in a declaration statement is instantiated.

We say that an analytical query $(A, P)$ is well-formed if:

- It does not have a conflict, and

- For every constraint statement $C_{i}$ and expression $e_{i}$ in the declaration statement of the form $T_{i} x_{i}=e_{i}$ or $\min x_{i}$, max $x_{i}$, or sat in flat $(A, P)$, the following holds: it only contains variables that have been declared in a declaration statement earlier in the sequence.

- If $A$ is a non-optimization sequence, then, flat $(A, P)$ must be data instantiated.

- If $A$ is an optimization sequence, then for every statement $S_{i}$ in flat $(A, P)=\left(s_{1}, \ldots, s_{n}\right)$ of the form $T_{i}$ $x_{i}=\ldots, x_{i}$ must be instantiated in $\left(S_{1}, \ldots, S_{i-1}\right)$ (i.e., earlier in the sequence).

From now on, only well-formed analytical queries are considered.

As discussed earlier, a SPAF model is an analytical model $M(I d)$ if it is one of the following forms:

- Process model

- Context model

- Flow model

- Flow aggregator model

- Sustainability metric aggregator model

A SPAF process model with identifier Id, denoted PM (Id), is an analytical sequence that contains statements of the following forms:

$$
\begin{array}{ll}
\text { string } & \text { Id.processType = type_flow_string, } \\
\{\text { string }\} & \text { Id.inputFlow = inputFlowExpr, } \\
\{\text { string }\} & \text { Id.outputFlow = outputFlowExpr, } \\
\{\text { string }\} & \text { Id.subProcess = subProcessExpr, } \\
\{\text { string }\} & \text { Id.flowAggregator = flowAggrExpr, }
\end{array}
$$

and where:

include $M(I)$, for every I in Id.inputFlow, Id.outputFlow, Id.subProcess, or Id.flowAggregator,

- Id is used as a prefix for all variables on the left hand side of the declaration statements, except for variables that appear on the left hand side of assignments into variables defined in the included 
models, i.e., SPAF models $M$ (Id'), where Id' is in Id.inputFlow, Id.outputFlow, Id.subProcess, and Id.flowAggregator (those are "visible" to the process model)

- type_process_string is a string

- inputFlowExpr, outputFlowExpr are analytical expressions of the type \{string\} (i.e., return a set of strings)

- subProcessIdsExpr, flowAggrIdsExpr are analytical expressions of type \{string\}

- $\quad M(I)$ denotes a method that returns a SPAF model with identifier $I$

A SPAF context model CM ( ), is an analytical model.

A SPAF flow model with identifier Id, denoted FM (Id), is an analytical model that contains statements of all of the following forms:

string Id.flowType = type_flow_string,

where:

- Id is used as a prefix for all variables on the left hand side of the assignment statements

- type_flow_string is a string

A SPAF flow aggregator model with identifier Id, denoted FAM (Id), is an analytical sequence that contains all of the followings forms:

$$
\begin{array}{ll}
\text { string } & \text { Id.flowType = type_flow_string, } \\
\text { \{string }\} & \text { Id.flows_to_aggr = inputFlowExpr, } \\
\text { \{string }\} & \text { Id.flows_from_aggr = outputFlowExpr, }
\end{array}
$$

where:

- Id is used as a prefix for all variables on the left hand side of the assignment statements

- type_flow_string is a string

- $\quad$ inputFlowExpr, outputFlowExpr are analytical expressions of the type \{string\} (i.e., return a set of strings)

An SPAF process package is a model package $P$. We say that it is well-formed if:

- $\quad P$ is closed under references

- $\quad P$ satisfies the following scoping rules:

- Process model $M(I d)$ can use variables prefixed with identifiers form Id.inputFlow, Id.outputFlow, Id.flowAggregator, or itself, i.e., Id.

- $\quad$ A model $M(I d)$ in $P$ can use variables from the context model in $P$.

- Flow Aggregator Model M(Id) can use variables that are prefixed with identifiers of flow models that are referenced in it, or itself, i.e., $I d$.

- For every process model $M$ in $P, A(M)$ is a well-formed analytical sequence

Note that a well-formed SPAF process package $P$ provides a modular description of a (flat) and wellformed analytical sequence. Thus, it is naturally extendable and its components are reusable.

\section{B. SPAF formal semantics}

We say that an analytical sequence $A$ is explicit if all of its analytical statements are of the form

$$
T_{i} \quad x_{i}=a_{i}
$$

where $a_{i}$ is a constant, i.e., it is an assignment of a constant to a variable. Intuitively, the symbolic expression of an explicit analytical sequence represents the corresponding data. Note that an explicit analytical sequence is flat. Formally, the semantics of an explicit analytical sequence $\left(s_{1}, \ldots, s_{n}\right)$, denoted $\operatorname{Sem}\left(\left(s_{1}, \ldots, s_{n}\right)\right)$, is itself, i.e., its symbolic expression. 
We say that an analytical sequence $A$ is implicit if all of its analytical statements are of the form

$$
T_{i} \quad x_{i}=e_{i}
$$

Note that this includes the case when the expression $e_{i}$ is a constant $a_{i}$. Formally, the semantics of a wellformed implicit analytical sequence

$$
\left(T_{1} \quad x_{1}=e_{1}, \ldots, T_{n} \quad x_{n}=e_{n}\right)
$$

is the explicit analytical sequence

$$
\left(\begin{array}{ll}
T_{1} & x_{1}=a_{1}, \ldots, T_{n} \quad x_{n}=a_{n}
\end{array}\right)
$$

in which each $a_{i}, l \leq i \leq n$, is a constant of type $T_{i}$ that is computed by expression $e_{i}$, when each variable $x_{j}, 1 \leq j \leq i-1$, is replaced by the constant $a_{j}$.

The semantics of $\left(s_{1}, \ldots, s_{n}\right)$ is denoted $\operatorname{Sem}\left(\left(s_{1}, \ldots, s_{n}\right)\right)$. Obviously, an explicit analytical sequence is a particular case of implicit, in which case, explicit and implicit semantics coincide.

We say that an analytical sequence $A=\left(s_{1}, \ldots, s_{n}\right)$ is a constraint analytical sequence if all of its statements are of the form

$$
\left(T_{i} x_{i}=e_{i}\right) \text { or } C_{i}
$$

where $e_{i}$ is an expression of type $T_{i}$ and $C_{i}$ is a constraint. Formally, the semantics of a well-formed constraint analytical sequence $\left(s_{1}, \ldots, s_{n}\right)$, denoted $\operatorname{Sem}\left(\left(s_{1}, \ldots, s_{n}\right)\right)$, is defined as follows:

- Consider an implicit analytical sequence $\left(S_{i_{1}}, \ldots, S_{i_{k}}\right)$, which is a sub-sequence of $\left(s_{1}, \ldots, s_{n}\right)$ that contains all statements $s_{i}$ ' of the form $T_{i} x_{i}=e_{i}$, and its semantics $\left(T_{i_{1}} x_{i_{1}}=a_{i_{1}}, \ldots, T_{i_{k}} x_{i_{k}}=a_{i_{k}}\right)$ (which is an explicit analytical sequence), and

- Consider a sequence $\left(C_{j_{1}}, \ldots, C_{j_{m}}\right)$, which is a sub-sequence of $\left(s_{1}, \ldots, s_{n}\right)$ that contains all the constraint statements

- If there exists $1 \leq i \leq m$, such that $C_{j_{i}}$ evaluates to FALSE after every variable $x_{i}$ in it is replaced with the constant $a_{i}$, then $\operatorname{Sem}\left(\left(s_{1}, \ldots, s_{n}\right)\right)$ is defined as INVALID. Otherwise, $\operatorname{Sem}\left(\left(s_{1}, \ldots, s_{n}\right)\right)$ is defined as the explicit analytical sequence $\left(T_{i_{1}} x_{i_{1}}=a_{i_{1}}, \ldots, T_{i_{k}} x_{i_{k}}=a_{i_{k}}\right)$.

We say that an analytical sequence $A=\left(s_{1}, \ldots, s_{n}\right)$ is an alternative analytical sequence, if each $s_{i}, 1 \leq i \leq$ $n$, is of the form

$$
\left(\begin{array}{ll}
\text { Ti } & x_{i}
\end{array}\right), \quad\left(T_{i} x_{i}=a_{i}\right), \quad\left(\begin{array}{ll}
T_{i} & x_{i}=e_{i}
\end{array}\right), \quad \text { or } C_{i}
$$

where $a_{i}$ is a constant of type $T i$, and $e_{i}$ is an expression of type $T i$, and $C_{i}$ is a constraint. Note that an alternative analytical sequence may have repetition of declaration statements for the same variable $x$. Consider the analytical sequence $\left(s_{1}, \ldots, s_{n}\right)$ resulting from $A$ by removing, for every variable $x$, all declarations except for its first appearance in A. Formally, the semantics of a well-formed alternatives analytical sequence $\left(s_{1}, \ldots, s_{n}\right)$, denoted $\operatorname{Sem}\left(\left(s_{1}, \ldots, s_{n}\right)\right)$, is defined as follows:

Consider all non-instantiated variables $x_{i_{1}}, \ldots, x_{i_{k}}$ in $\left(s_{1}, \ldots, s_{n}\right)$. Sem $\left(\left(s_{1}, \ldots, s_{n}\right)\right)$ is the set

$$
\left\{E\left(a_{i_{1}}, \ldots, a_{i_{k}}\right) \mid a_{i_{1}} \text { in } D\left(T_{i_{1}}\right), \ldots, a_{i_{k}} \text { in } D\left(T_{i_{k}}\right) \wedge E\left(a_{i_{1}}, \ldots, a_{i_{k}}\right) \neq I N V A L I D\right\}
$$

where:

- $D\left(T_{i_{1}}\right), \ldots, D\left(T_{i_{k}}\right)$ are the domains of types $T_{i_{1}}, \ldots, T_{i_{k}}$, respectively, and 
- $E\left(a_{i_{1}}, \ldots, a_{i_{k}}\right)$ denotes Sem $\left(\left(s_{1}, \ldots, s_{n}\right)\left[x_{i_{1}} / a_{i_{1}}, \ldots, x_{i_{k}} / a_{i_{k}}\right]\right)$, where $\left(s_{1}, \ldots, s_{n}\right)\left[x_{i_{1}} / a_{i_{1}}, \ldots, x_{i_{k}} / a_{i_{k}}\right]$ denotes the constraint analytical sequence $\left(s_{1}, \ldots, s_{n}{ }^{\prime}\right)$ that results from $\left(s_{1}, \ldots, s_{n}\right)$ by replacing each statement of the form $\left(T_{i_{j}} x_{\boldsymbol{i}_{j}}\right), 1 \leq j \leq k$, with the statement $T_{i_{j}} x_{i_{j}}=a_{i_{j}}$.

We say that an analytical sequence $A=\left(s_{1}, \ldots, s_{n}, s_{n+1}\right)$ is a flat optimization sequence if $\left(s_{1}, \ldots, s_{n}\right)$ is an alternative sequence, and $s_{(n+1)}$ is of the form:

$\min x_{i}, \quad \max x_{i}$, or sat.

where $x_{i}, l \leq i \leq n$, is one of the variables in the left hand sides of assignments in

$\left(s_{1}, \ldots, s_{n}\right)$. Assuming without loss generality that, for every variable $x$ in $A$, there is a single declaration of $x$ (if this is not the case, all declarations of $x$ except for its first appearance are removed.) Formally, the semantics of an optimization analytical sequence $\left(s_{1}, \ldots, s_{n}, s_{(n+1)}\right)$, denoted $\operatorname{Sem}\left(\left(s_{1}, \ldots, s_{n}, s_{(n+1)}\right)\right)$, is defined as follows:

If $\operatorname{Sem}\left(s_{1}, \ldots, s_{n}\right)=\emptyset$ then we say that $\operatorname{Sem}\left(\left(s_{1}, \ldots, s_{n}, s_{(n+1)}\right)\right)$ is INFEASIBLE. Otherwise, consider an explicit analytical sequence $E$ in $\operatorname{Sem}\left(\left(s_{1}, \ldots, s_{n}\right)\right)$ such that:

- If $s_{(n+1)}$ is $\min x_{i}$, then for all $E$ ' in Sem $\left(\left(s_{1}, \ldots, s_{n}\right), a_{i} \leq a_{i}\right.$, where $a_{i}$ and $a_{i}$ ', are the analytical model constants in the assignments $T_{i} x_{i}=a_{i}$ of $E$, and $T_{i} x_{i}=a_{i}^{\prime}$ ' of $E$ '.

- If $s_{(n+1)}$ is $\max x_{i}$, then for all $E^{\prime}$ in $\operatorname{Sem}\left(\left(s_{1}, \ldots, s_{n}\right), a_{i} \geq a_{i}\right.$, where $a_{i}$ and $a_{i}{ }^{\prime}$, are the analytical model constants in the assignments $T_{i} x_{i}=a_{i}$ of $E$, and $T_{i} x_{i}=a_{i}^{\prime}$ of $E$ '.

If $E$ does not exist, we say that $\operatorname{Sem}\left(\left(s_{1}, \ldots, s_{n}, s_{(n+1)}\right)\right)$ is UNBOUNDED. Otherwise,

$\operatorname{Sem}\left(\left(s_{1}, \ldots, s_{n}, s_{(n+1)}\right)\right)$ is $E$.

Note that if $s_{(n+1)}$ is sat, the semantics is just an explicit analytical sequence $E$ in $\operatorname{Sem}\left(\left(s_{1}, \ldots, s_{n}\right)\right)$. Also note that the optimization semantics (whether it is minimization, maximization, or satisfiability) are non-deterministic, i.e., there may be more than one explicit model that satisfies the condition in the definition of semantics.

Consider the five layers (types) of analytical sequences (1) explicit, (2) implicit, (3) constraints, (4) alternatives, and (5) optimization. Let $L$ (1), $L$ (2), $L$ (3), $L$ (4), and $L$ (5) denote sets of analytical sequences that can be expressed by each layer, respectively. We claim that

$$
L(1) \subset L(2) \subset L(3) \subset\{L(4)\} \subset L(5)
$$

and that the semantics of each layer are consistent with all lower layers. That is, for any two layers $i, j, 1$ $\leq i<j \leq 5, i \neq 4, j \neq 4$, if an AM $A$ is in $L(i)$, then $\operatorname{Sem}(i)$ of $A$ is also Sem (j) of $A$.

Semantics of a query $(A, P)$ is a pair $\left(A^{\prime}, P^{\prime}\right)$ constructed as follows:

- For every sequence $S$, either $A$ or a sequence $B$ in a model $M(I d)\{B\}$ in $P, S$ is replaced by $S$ ' as follows.

- Consider all variables $x_{1}, \ldots, x_{n}$, declared in their order in $S$, then $S$ ' is the sequence

$$
\left(\begin{array}{lllll}
T_{1} & x_{1}=a_{1}, \ldots, T_{n} & x_{n}=a_{n}
\end{array}\right)
$$

where $T_{1}, \ldots, T_{n}$ are the corresponding types of $x_{1}, \ldots, x_{n}$ respectively, and $a_{i}$ is the constant instantiated with $x_{i}$ in the semantics $E$ of flat $(A, P)$.

\section{SPAF Query Computation}

In this section, algorithms (reduction procedures) to perform SPAF analytical query computation are introduced. Figure C.1 shows a commutative diagram for analytical query computation, in which the upper left box indicates the query sequence $A$ in model package $P$. The query sequence may have include statements. The semantics of $A$ is sequence $A$ ' in package $P$ ' as shown in the upper right box in Figure 
24. Two algorithms are included in the computation - analytical query algorithm and flat optimization sequence algorithm. Through the analytical query algorithm (refer to step (1), (6), and (5)), $(A, P)$ can be translated to a flat analytical sequence (middle left box). If the flat analytical sequence can be instantiated, it is an implicit analytical sequence, otherwise, it is an optimization analytical sequence whose semantics is a flat explicit analytical sequence (middle right box). This algorithm calls the flat optimization sequence algorithm (refer to step (2), (3), and (4)) to translate the flat optimization sequence to a standard optimization model such as OPL or AMPL (lower left box). By using an optimization solver, the optimization solution (lower right box) can be derived. All variables can then be instantiated, the sequence becomes a flat explicit analytical sequence (middle right box), which can be translate back to $\left(A^{\prime}, P^{\prime}\right)$.

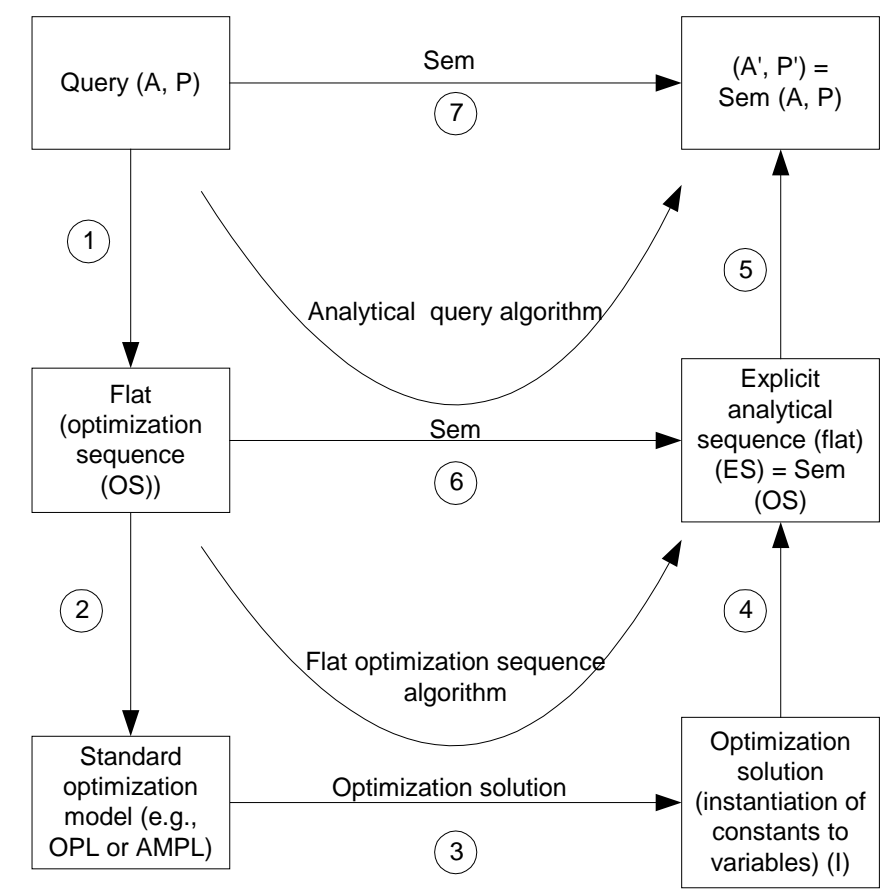

Figure C.1 A commutative diagram for analytical query computation

Figure C.2 presents the algorithm of SPAF Query Computation. The input is an analytical query sequence $A$ and a model package $P$ that is closed with respect to $A$. The output is ( $\left.A^{\prime}, P^{\prime}\right)$ that is the semantics of ( $A$, $P)$. The procedures of the algorithm include:

1. Construct a flat sequence $S 1$ by replacing all the include statements in $A$ with corresponding analytical sequences.

2. Construct a new sequence $S 2$ by removing all the duplicated declarations of $x$ except for the first declaration in $S 1$ for every variable $x$ declared in $S 1$.

3. If $S 2$ is instantiated, it must be an implicit analytical sequence. So a new explicit analytical sequence $S 3$ can be constructed by replacing each variable with a constant that derived from an expression.

4. If $S 2$ is not instantiated, it must be a flat optimization query. By calling the OptSeqAlg (S2) algorithm, it will return the semantics of $S 2$. 
Input: $(A, P)$ is a well-formed analytical query and $P$ is a closed form model package closed with respect to $A$.

Output: $\left(A^{\prime}, P^{\prime}\right)$ is the semantics of $(A, P)$.

1. Construct $S 1=$ flat $(A, P)$.

2. Construct sequence S2 from S1 as follows:

For every variable $x$ declared in S1, remove all declarations of $x$

except for the first declaration in 51.

3. Check if 52 is instantiated.

4. If $S 2$ is instantiated, it must be an implicit analytical sequence of the form $\left(T_{1}\right.$ $\left.x_{1}=e_{1}, \ldots, T_{n} x_{n}=e_{n}\right)$. In this case, construct $S 3$ as the explicit analytical sequence $\left(T_{1} x_{1}=a_{1}, \ldots, T_{n} x_{n}=a_{n}\right)$, in which each $a_{i}, 1 \leq i \leq n$, is a constant of type $T_{i}$ that is computed by expression $e_{i}$, where each variable $x_{j}, 1 \leq j \leq i-1$, is replaced by $a_{j}$.

5. Otherwise, if $S 2$ is not instantiated, it must be a flat optimization query. Construct S3 by calling the method OptSeqALg (S2), which returns the semantics of S2.

6. Construct the pair ( $\left.A^{\prime}, P^{\prime}\right)$ as follows:

For every sequence $S$, which is either $A$ or a sequence $B$ in $a$ model(Id) $\{B\}$ in $P$, $S$ is replaced by $S$ ' as follows. Consider all variables $x_{1}, \ldots, x_{n}$, declared in their order in $S$, then $S$ ' is the sequence $\left(T_{1} x_{1}=a_{1}, \ldots, T_{n} x_{n}=a_{n}\right)$ where $T_{1}, \ldots, T_{n}$ are the corresponding types of $x_{1}, \ldots, x_{n}$ respectively, and $a_{i}$ is the constant to instantiate $x_{i}$ in 53 .

\section{Figure C.2 Algorithm 1: SPAF query computation}

Figure C.3 presents the algorithm of Optimization Sequence Algorithm (OptSeqAlg). The input is a flat optimization query S2 generated by the SPAF Query Computation algorithm. The output is the semantics of $S 2$.

1. For all variables that are instantiated in every statement, replace the expression with the computed constant.

2. Construct decision variables that are not being instantiated.

3. Construct a set of constraints by replacing decision variables in every statement with its constant. For any variable that is non-instantiated, a constraint is added.

4. Construct the optimization problem with objectives and constraints. 
5. Solve the optimization problem using an optimization solver

6. Construct the answer sequence by removing all constraint statements and replacing all the variables using constants computed or the optimization solutions.

Input: Flat optimization query (i.e., $\left(s_{1}, \ldots, s_{n}, s_{n+1}\right)$ where $s_{n+1}$ is of the

form $\min x_{i}, \max x_{i}(1 \leq i \leq n)$ or sat where $x_{i}$ is not

instantiated on $\left.\left(s_{1}, \ldots, s_{n}\right)\right)$.

Output: Semantics of $\left(s_{1}, \ldots, s_{n}, s_{n+1}\right)$.

1. Consider all variables $x_{i_{1}}, \ldots, x_{i_{m}}$ in $\left(s_{1}, \ldots, s_{n}\right)$ that are instantiated. For every statement $S_{i_{j}}, 1 \leq j \leq k$, of the form

$T_{i_{j}} x_{i_{j}}=e_{i_{j}}$, compute $e_{i_{j}}$, and replace $e_{i_{j}}$ with the computed constant

$a_{i_{j}}$, i.e., resulting in $T_{i_{j}} x_{i_{j}}=a_{i_{j}}$

2. Construct the set of decision variable $v$ to be the set of all non-instantiated variables $x_{l_{1}}, \ldots, x_{l_{m}}$ in $\left(s_{1}, \ldots, s_{n}\right)$ ranging over the domains corresponding to types $T_{l_{1}}, \ldots, T_{l_{m}}$ respectively.

3. Construct the set of constraints $C$ as follows:

3.1 Initially, $C=\varnothing$.

3.2 For every statement $s_{i}, 1 \leq i \leq n$ of the form $C_{i}$, add to $C$ the constraint resulting from $C_{i}$ by replacing every instantiated variable $x_{i_{j}}$ with its constant $a_{i_{j}}$ from step 1 .

3.3 For every statement $S_{i}$ of the form $T_{i} x_{i}=e_{i}$, where $x_{i}$ is noninstantiated, add the constraint $x_{i}==e_{i}$ ', where $e_{i}$ ' result from $e_{i}$ by replacing each decision variable $x_{i_{j}}$ in $e_{i}$ with its constant $a_{i_{j}}$ from step 1 .

4. Construct the optimization problem 0 ;

$\min _{V} x_{n}$ subject to $C, \max _{V} x_{n}$ subject to $C$, or $\operatorname{sat}_{V} C$ according to $s_{n+1}$.

5. Solve the optimization problem 0 .

6. If $O$ is infeasible, return "INFEASIBLE", else if $O$ is unbounded, return "UNBOUNDED." 
7. Otherwise, construct the answer sequence from $\left(s_{1}, \ldots, s_{n}\right)$ as follows:

7.1 ALL non-declaration statements (i.e., constraints) are removed.

7.2 Every declaration statement with type $T_{i}$ and variable $x_{i}$ (i.e.,

of the form $T_{i} \quad x_{i}=a_{i}$ or $T_{i} x_{i}=e_{i}$ ) be replaced as follows:

7.3 if $x_{i}$ is instantiated, it is replaced with $T_{i} x_{i}=a_{i}$, where $a_{i}$

is a constant computed in Step 1.

7.4 if $x_{i}$ is non-instantiated, the statement is replaced with

$T_{i} x_{i}=a_{i}$, where $a_{i}$ is a constant instantiated into decision

variable $x_{i}$ from the solution of the optimization problem 0 .

Figure C.3 Algorithm 2: optimization sequence algorithm (OptSeqAlg)

Algorithm correctness: We denote by All-Sem (A, P) the set of all explicit analytical sequences' E that are Sem (A, P).

We denote by All-Ans (A, P) the set of all explicit analytical sequences' $\mathrm{E}$ that are possible answers produced by Algorithm: SPAF query computation.

Claim: Algorithm SPAF query computation is CORRECT, i.e., it is:

1. Sound, i.e., for every well-formed analytical query (A, P),

$$
\text { All_Ans }(\mathrm{A}, \mathrm{P}) \subseteq \text { All_Sem }(\mathrm{A}, \mathrm{P})
$$

2. Complete, i.e., for every well-formed analytical query (A, P),

All_Sem (A, P) $\subseteq$ All_Ans (A, P) 